Review

\title{
Optimized Energy Management Schemes for Electric Vehicle Applications: A Bibliometric Analysis towards Future Trends
}

\author{
Md. Sazal Miah ${ }^{1}\left(\mathbb{D}\right.$, Molla Shahadat Hossain Lipu ${ }^{2,3, *} \mathbb{D}^{\circ}$, Sheikh Tanzim Meraj ${ }^{4}\left(\mathbb{0}\right.$, Kamrul Hasan $^{5}{ }^{(0)}$,

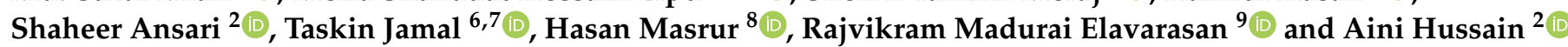 \\ 1 School of Engineering and Technology, Asian Institute of Technology, Pathumthani 12120, Thailand; \\ st121577@ait.asia \\ 2 Department of Electrical, Electronic and Systems Engineering, Universiti Kebangsaan Malaysia, \\ Bangi 43600, Selangor, Malaysia; p100855@siswa.ukm.edu.my (S.A.); draini@ukm.edu.my (A.H.) \\ 3 Center for Automotive Research (CAR), Universiti Kebangsaan Malaysia, Bangi 43600, Selangor, Malaysia \\ 4 Department of Electrical and Electronic Engineering, Universiti Teknologi PETRONAS, \\ Seri Iskandar 32610, Perak, Malaysia; sheikh_19001724@utp.edu.my \\ 5 School of Electrical Engineering, College of Engineering Studies, Universiti Teknologi MARA, \\ Shah Alam 40450, Selangor, Malaysia; 2019984679@isiswa.uitm.edu.my \\ 6 Department of Electrical and Electronic Engineering, Ahsanullah University of Science and Technology, \\ Dhaka 1208, Bangladesh; taskinjamal@gmail.com \\ 7 School of Geography, Geology and the Environment, Keele University, Staffordshire ST5 5BG, UK \\ 8 Faculty of Engineering, University of the Ryukyus, 1 Senbaru, Okinawa 903-0213, Japan; \\ k198676@eve.u-ryukyu.ac.jp \\ 9 Department of Electrical and Electronics Engineering, Thiagarajar College of Engineering, \\ check for \\ Madurai 625015, India; rajvikram787@gmail.com \\ * Correspondence: lipu@ukm.edu.my
}

Citation: Miah, M.S.; Hossain Lipu, M.S.; Meraj, S.T.; Hasan, K.; Ansari, S.; Jamal, T.; Masrur, H.; Elavarasan, R.M.; Hussain, A. Optimized Energy Management Schemes for Electric Vehicle Applications: A Bibliometric Analysis towards Future Trends. Sustainability 2021, 13, 12800. https:/ / doi.org/10.3390/su132212800

Academic Editor: Alberto-Jesus Perea-Moreno

Received: 1 November 2021

Accepted: 15 November 2021

Published: 19 November 2021

Publisher's Note: MDPI stays neutral with regard to jurisdictional claims in published maps and institutional affiliations.

Copyright: (c) 2021 by the authors. Licensee MDPI, Basel, Switzerland. This article is an open access article distributed under the terms and conditions of the Creative Commons Attribution (CC BY) license (https:/ / creativecommons.org/licenses/by/ $4.0 /)$.
Abstract: Concerns over growing greenhouse gas (GHG) emissions and fuel prices have prompted researchers to look into alternative energy sources, notably in the transportation sector, accounting for more than $70 \%$ of carbon emissions. An increasing amount of research on electric vehicles (EVs) and their energy management schemes (EMSs) has been undertaken extensively in recent years to address these concerns. This article aims to offer a bibliometric analysis and investigation of optimized EMSs for EV applications. Hundreds (100) of the most relevant and highly influential manuscripts on EMSs for EV applications are explored and examined utilizing the Scopus database under predetermined parameters to identify the most impacting articles in this specific field of research. This bibliometric analysis provides a survey on EMSs related to EV applications focusing on the different battery storages, models, algorithms, frameworks, optimizations, converters, controllers, and power transmission systems. According to the findings, more articles were published in 2020, with a total of 22, as compared to other years. The authors with the highest number of manuscripts come from four nations, including China, the United States, France, and the United Kingdom, and five research institutions, with these nations and institutions accounting for the publication of 72 papers. According to the comprehensive review, the current technologies are more or less capable of performing effectively; nevertheless, dependability and intelligent systems are still lacking. Therefore, this study highlights the existing difficulties and challenges related to EMSs for EV applications and some brief ideas, discussions, and potential suggestions for future research. This bibliometric research could be helpful to EV engineers and to automobile industries in terms of the development of cost-effective, longer-lasting, hydrogen-compatible electrical interfaces and well-performing EMSs for sustainable EV operations.

Keywords: energy management; optimization; converter; controller; battery storage; electric vehicle

\section{Introduction}

The demand for urban mobility is rapidly increasing [1]. $\mathrm{CO}_{2}$ concentrations in 2012 were roughly $40 \%$ higher than in the mid-1800s, based on the International En- 
ergy Agency [2,3]. Electric vehicles (EVs) offer significant promise in terms of reducing transportation-related energy and emissions [4,5]. Due to the growing concerns over global warming, the development of $\mathrm{EV}$ applications has recently received much attention because of its benefits in decreasing $\mathrm{CO}_{2}$ and GHG. EVs require a low-emission electric motor and advanced power electronics technology as well as improved energy management methods for energy sources and storage systems such as fuel cells (FCs), supercapacitors (SCs), and batteries to achieve adequate driving performances [6-9]. Energy management schemes (EMSs) benefit EVs by improving reliability, flexibility, and power quality [10]. To meet transit power supply and demand, the development of EVs with adequate energy and power density to achieve suitable driving performances, and the connection of FC sources to SC storage systems, is critical [11]. When sufficient fuel (gases and hydrogen) is available, FCs sources can provide an uninterruptible power supply. Due to the time responsiveness of the gas supply system, these energy sources can have a relatively slow transient dynamic. On the other hand, supercapacitor energy storage systems may provide high instantaneous power for short periods but have a lower energy density than other traditional storage elements such as batteries [12-14].

In the literature, many EMSs for EV applications have been described [15-17]. Optimization, filters, controllers, and rule-based techniques are the four types of methods that can be categorized. Neural Networks, Fuzzy Logic, and State Machines are the most common rule-based methods $[16,18]$. Each rule or state is defined either heuristically or experimentally for State Machine control [16]. Furthermore, Fuzzy Logic rule-based techniques attach membership functions to the inputs and outputs to attain the necessary performance. The performance of rule-based techniques is linked to the system's knowledge. The basic idea of controller-based EMSs is to employ control rules to correct the error between desired and actual states. Backstepping control [19], Sliding control [20], H-infinity control [21], Passivity control [22], Flatness-based control [23], ProportionalIntegral (PI) control [24], and so on are examples of energy management-based controllers. Even when the operating point is unknown, these approaches can precisely estimate the reference while accounting for system losses. Filter techniques use a frequency decoupling strategy that considers the energy system's dynamic properties and physical features. Fast Fourier Transform methods [25], the Wavelet technique [26], and Low-Pass filter methods [27] are mostly used to accomplish this. When the system frequencies are accurately determined, the filter-based management method is straightforward and may greatly increase the lifetime of EV applications. Optimization-based approaches have recently been investigated for dealing with complicated management objectives (lifetime, efficiency, cost, etc.). The desired references are obtained via minimizing an instantaneous cost function in such techniques. Particle Swarm Optimization [28], Optimal Power Distribution Control [29], Adaptive Optimal Control [30], Neural Networks [24], Stochastic Dynamic Programming [31], and Model Predictive Control [32] are some of the techniques mentioned in the literature. These techniques are complicated and involve many calculations, slowing down the energy management system's response time.

Bibliometrics is a research strategy that uses library and information science to offer information and analysis in various formats, such as statistics and quantitative approaches [33,34]. Bibliometrics is a vital research topic because it provides specific and historical data that may be utilized to forecast future research trends [35,36]. Universities, instructors, researchers, and professors can use bibliometric studies to assess the quality of research using a variety of important indicators such as h-indexes, impact factors, citations, and current standing. Gingras [37] addressed the influence of bibliometric analysis on research direction and proposed some criteria for developing a suitable assessment procedure at a specific size of research plan and analysis. Andres [38] detailed the steps for conducting bibliometric analysis with many real-world samples and interpreted the results. In the bibliometric study, the authors also explained the significance of Scientometric investigations. The current condition of publishing activity in EMSs for EV application is examined in this article using a bibliographic analysis. Numerous bibliometric techniques 
have been used in recent years to assess the research progress in various fields, such as healthcare simulation [39], Pediatric Surgery [40], drug repurposing [41], technological innovation [42], Strategic Management [43], Industrial Ecology [44], climate engineering research [45], applications of artificial intelligence [46], Computers and Industrial Engineering [47], quantum electronics [48], engineering nanomaterials [49], and software engineering [50].

There are a couple of bibliometric analyses performed on the EV applications related topics such as life cycle cost analysis for EVs [51], bibliometric analysis on EVs [52], EV reliability [53], next-generation vehicles [54], fuzzy optimization-based EVs energy technologies [55], carbon emissions from the transport sector [56], autonomous vehicles [57], and the development of China's EV battery industry [58]. As per our knowledge, no bibliometric analysis on EMSs for EV applications has been conducted so far. As a result, this paper outlines the first bibliometrics analysis of EMSs for EVs, which was carried out during the last eleven years (from 2010 to September 2021) to examine the assessments, research community, and current developments in this area. The following findings emphasize the paper's key contributions.

- A brief summary of EMSs for EV applications is presented regarding the number of articles published to date. The analysis is carried out on a yearly basis, subsequently includes a discussion.

- The most prolific authors, the most productive university, and the nation dominating the publishing are all used to analyze EMSs for EV.

- The keywords and themes that were utilized for content analysis and gap analysis are evaluated.

- Publication document types such as original papers, systematic and non-systematic reviews, and book chapters are investigated. In addition, the journals' impact factors and publisher distributions are investigated.

- The amount of researcher collaboration is determined. The number of authors in the articles and the connection between diverse universities and nations are also used to assess the team.

- The most influential authors, universities, institutions, and nations with the most published research are identified. This is critical for determining the productivity of authors, organizations, and nations in the research sector and improving research output and collaboration among authors.

The bibliometric review aims to find the top 100 most relevant publications in the field of EMSs for EV applications. As a result, a comprehensive report on these publications facts, critical debates, analyses, contributions, and flaws is provided. The following are some of the advantages that the article will provide.

- A better understanding of the history and evolution of EMSs for EV applications will be available to future researchers.

- A comparative analysis of the most relevant articles for EMS in the EV applications field, which will aid in the future construction of existing knowledge and practice, will be given.

- Finally, this bibliometric analysis will include fruitful recommendations for the prospects and developments of EMSs for EV applications.

This bibliometric analysis is arranged as follows: Section 1 offers an overview of the EMSs, bibliometric study, research gaps, and contributions; Section 2 presents a detailed overview of the surveying methodologies used in bibliometric analysis; and Section 3 presents a comprehensive analysis on the selected papers on EMSs for EV applications. Section 4 discusses the various issues, challenges, and problems. The future trends and recommendations are highlighted in Section 5, followed by conclusions in Section 6. 


\section{Surveying Methods}

A statistical, bibliometric study of the Scopus database (www.scopus.com), was utilized to conduct this research. Since it contains a higher number of articles than other databases, such as Web of Science [59], the Scopus database was chosen as a source in this study's bibliometric analysis. Due to the lack of reliable results, Google Scholar was not evaluated in this study [60]. The "energy management schemes" study was recorded in the Scopus database at the end of September 2021. Figure 1 depicts the bibliometric analysis methodologies employed in the Scopus database. As indicated in the picture below, the procedure was divided into six stages:

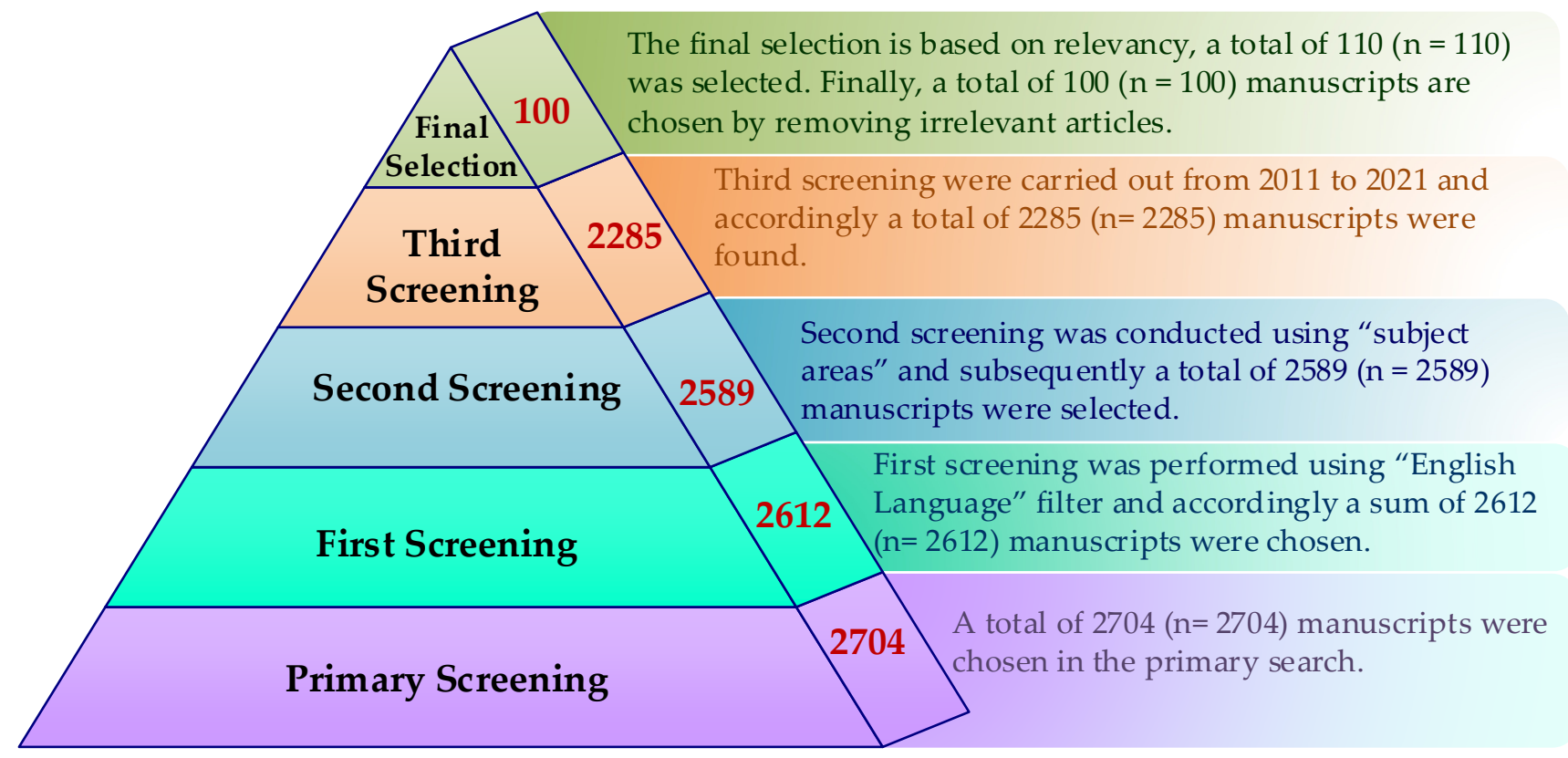

Figure 1. Manuscript selection steps from Scopus database.

\subsection{Selection and Exclusion Criteria}

Some predefined criteria were used to select the articles from a specific Scopus database. Table 1 represents the primary search keyword codes used for the article search of the Scopus database. The following are the criteria for article inclusion and exclusion for the 100 most relevant manuscripts in the field of EMSs for EV applications:

- The primary criteria for including manuscripts were the following keywords: energy management system, converter, controllers, optimization, and EVs. Some articles were excluded from this list based on the irrelevancy of the field.

- For the objectives of the study, articles published in the English language between 2012 and 2021 were examined.

Table 1. Keyword codes used to search for potential manuscripts in the Scopus database.

\begin{tabular}{cccc}
\hline Stages & Filter & Keyword Codes & $\begin{array}{c}\text { Number of } \\
\text { Manuscripts }\end{array}$ \\
\hline \multirow{2}{*}{ 1st stage } & $\begin{array}{c}\text { Energy Management system, } \\
\text { Electric vehicle applications }\end{array}$ & $\begin{array}{c}\text { TITLE-ABS-KEY (energy AND management AND system } \\
\text { AND for AND electric AND vehicle AND applications) }\end{array}$ & 2704 \\
\hline \multirow{2}{*}{ 2nd stage } & English & TITLE-ABS-KEY (energy AND management AND system & AND for AND electric AND vehicle AND applications) \\
& & AND (LIMIT-TO (LANGUAGE, “English”)) & 2612 \\
\hline
\end{tabular}


Table 1. Cont.

\begin{tabular}{|c|c|c|c|}
\hline Stages & Filter & Keyword Codes & $\begin{array}{l}\text { Number of } \\
\text { Manuscripts }\end{array}$ \\
\hline 3rd stage & Subject area & $\begin{array}{l}\text { TITLE-ABS-KEY (energy AND management AND system } \\
\text { AND for AND electric AND vehicle AND applications) } \\
\text { AND (LIMIT-TO (LANGUAGE, “English")) AND } \\
\text { (LIMIT-TO (SUBJAREA, “ENGI") OR LIMIT-TO } \\
\text { (SUBJAREA, “ENER") OR LIMIT-TO (SUBJAREA, } \\
\text { “COMP") OR LIMIT-TO (SUBJAREA, “MATH”) OR } \\
\text { LIMIT-TO (SUBJAREA, “ENVI") OR LIMIT-TO } \\
\text { (SUBJAREA, “PHYS") OR LIMIT-TO (SUBJAREA, “MATE”) } \\
\text { OR LIMIT-TO (SUBJAREA, “CHEM")) }\end{array}$ & 2589 \\
\hline 4th stage & Year range (2010-2021) & $\begin{array}{l}\text { TITLE-ABS-KEY (energy AND management AND system } \\
\text { AND for AND electric AND vehicle AND applications) } \\
\text { AND (LIMIT-TO (LANGUAGE, “English”)) AND } \\
\text { (LIMIT-TO (SUBJAREA, “ENGI”) OR LIMIT-TO } \\
\text { (SUBJAREA, “ENER”) OR LIMIT-TO (SUBJAREA, } \\
\text { “COMP”) OR LIMIT-TO (SUBJAREA, “MATH”) OR } \\
\text { LIMIT-TO (SUBJAREA, “ENVI”) OR LIMIT-TO } \\
\text { (SUBJAREA, “PHY") OR LIMIT-TO (SUBJAREA, “MATE”) } \\
\text { OR LIMIT-TO (SUBJAREA, “CHEM”)) AND (LIMIT-TO } \\
\text { (PUBYEAR, 2022) OR LIMIT-TO (PUBYEAR, 2021) OR } \\
\text { LIMIT-TO (PUBYEAR, 2020) OR LIMIT-TO (PUBYEAR, } \\
\text { 2019) OR LIMIT-TO (PUBYEAR, 2018) OR LIMIT-TO } \\
\text { (PUBYEAR, 2017) OR LIMIT-TO (PUBYEAR, 2016) OR } \\
\text { LIMIT-TO (PUBYEAR, 2015) OR LIMIT-TO (PUBYEAR, } \\
\text { 2014) OR LIMIT-TO (PUBYEAR, 2013) OR LIMIT-TO } \\
\text { (PUBYEAR, 2012) OR LIMIT-TO (PUBYEAR, 2011) OR } \\
\text { LIMIT-TO (PUBYEAR, 2010)) }\end{array}$ & 2285 \\
\hline
\end{tabular}

\subsection{Screening Procedures}

As there are vast numbers of articles published in the various journals, the following criteria were applied to select the most relevant articles from the Scopus database.

- Based on the primary selection, a total of $2704(n=2704)$ articles were chosen.

- By applying "English Language", a sum of $2612(\mathrm{n}=2612)$ publications were filtered.

- Then, a total of $2589(\mathrm{n}=2589)$ manuscripts were selected by limiting the subject areas.

- After limiting the year ranges from 2010 to 2021, a total of 2285 articles were filtered.

- The final selection was based on relevancy; a sum of $110(n=110)$ was selected.

- After manually removing irrelevant articles, a total of $100(n=100)$ manuscripts from the Scopus database published in various journals were selected for the final evaluation.

\subsection{Research Trend}

Researchers are currently demonstrating interest in developing more efficient EMSs for EV applications $[6,61]$. They used a variety of approaches to find suitable EMSs for EV applications to ensure more efficient energy management. Figure 2 depicts the trend in research from 2010 to 2021. Overall, the number of papers produced every year due to the primary screening of the chosen database increased. Figure 2 shows that as the number of published papers rises, so does the number of researchers with related research interests. From 2019 through 2021, a total of 927 manuscripts were published. In comparison, there were 1774 papers published in the nine years leading up to 2018. In the first nine years of publication (2010-2018), 65\% of all papers were published, while papers produced between 2019 and 2021 account for 35\% of the total. According to the graph, the last three years show a linear growth of publications in EMSs related to EV applications. 


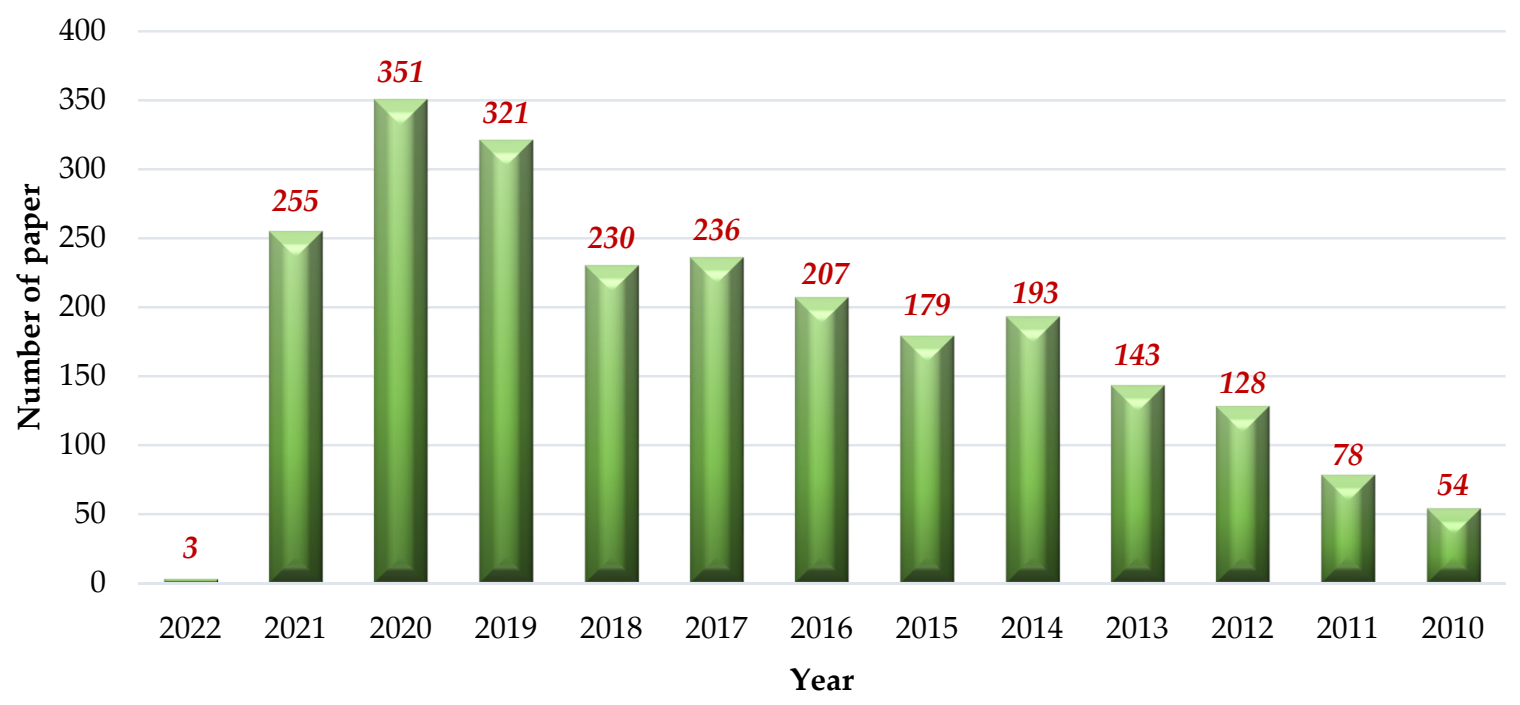

Figure 2. Manuscript distribution over the years from 2010 to 2022.

\subsection{Data Extraction}

By utilizing the Scopus database, information on manuscripts was extracted based on the following variables: names of the authors; doi of the manuscript; keywords list; year of publication; the name of the manuscript's publisher; type of manuscript; the name of the publication country based on the first author's affiliations; the total number of citations; the number of citations in the last five years. Following the data analysis from the chosen article, observations were made to present a clearer picture of EMSs for EV applications.

\subsection{Study Characteristics and Outcomes}

From the primary search, the Scopus database yielded a sum of 2704 manuscripts. By applying numerous filtering methods, the most relevant 100 articles were chosen and listed in Table 2 with the names of the authors; the doi of the manuscripts; the keywords lists; the years of publication; the names of the manuscripts' publishers; the types of the manuscripts; the names of the publication countries based on the firsts authors' affiliations; the total number of citations; and the total number of citations in the last five years. The total number of citations for the chosen manuscripts is 4903 (mean 49.52; median 14; and citation range 0 to 673). Furthermore, 11 of the 100 manuscripts were cited over 100 times.

Table 2. The most relevant 100 manuscripts in the field of EMSs for EV applications.

\begin{tabular}{|c|c|c|c|c|c|c|c|c|}
\hline Rank & Ref. no. & Keywords & $\begin{array}{l}\text { Type of } \\
\text { Article }\end{array}$ & $\begin{array}{l}\text { Abbreviated } \\
\text { Journal Name }\end{array}$ & $\begin{array}{l}\text { Publisher } \\
\text { Name }\end{array}$ & Year & Country & Citation \\
\hline 1 & {$[62]$} & BMS, EV, LIB, SOC & Review & RSERF & $\begin{array}{l}\text { Elsevier } \\
\text { Ltd }\end{array}$ & 2017 & Malaysia & 673 \\
\hline 2 & [63] & $\begin{array}{c}\text { BMS, BT, } \\
\text { Charge/discharge, } \\
\text { EV, SOC, SOH }\end{array}$ & Article & IEM & IEEE & 2013 & $\begin{array}{l}\text { United } \\
\text { States }\end{array}$ & 487 \\
\hline 3 & [6] & $\begin{array}{c}\text { EV, ESS, } \\
\text { Hybridization, } \\
\text { Power electronics }\end{array}$ & Review & RSERF & $\begin{array}{l}\text { Elsevier } \\
\text { Ltd }\end{array}$ & 2017 & Malaysia & 384 \\
\hline 4 & {$[64]$} & $\begin{array}{l}\text { EL, Forgetting factor, } \\
\text { Kullback-Leibler } \\
\text { divergence, PM, RL }\end{array}$ & Article & APEND & $\begin{array}{l}\text { Elsevier } \\
\text { Ltd }\end{array}$ & 2018 & China & 222 \\
\hline 5 & [65] & EV, EMS, LIB, SOC & Review & IEEE Access & IEEE & 2018 & Malaysia & 211 \\
\hline 6 & {$[66]$} & EV, EMS, HESS & Article & APEND & $\begin{array}{l}\text { Elsevier } \\
\text { Ltd }\end{array}$ & 2014 & China & 206 \\
\hline
\end{tabular}


Table 2. Cont.

\begin{tabular}{|c|c|c|c|c|c|c|c|c|}
\hline Rank & Ref. no. & Keywords & $\begin{array}{l}\text { Type of } \\
\text { Article }\end{array}$ & $\begin{array}{c}\text { Abbreviated } \\
\text { Journal Name }\end{array}$ & $\begin{array}{c}\text { Publisher } \\
\text { Name }\end{array}$ & Year & Country & Citation \\
\hline 7 & [67] & $\begin{array}{l}\text { CC, EV, LIB, } \\
\text { TD, TMS }\end{array}$ & Review & JPSOD & $\begin{array}{c}\text { Elsevier } \\
\text { B.V. }\end{array}$ & 2017 & China & 186 \\
\hline 8 & [68] & $\begin{array}{l}\text { EV, EM, ES, } \\
\text { Optimization, } \\
\text { Real-time }\end{array}$ & Article & APEND & $\begin{array}{l}\text { Elsevier } \\
\text { Ltd }\end{array}$ & 2016 & France & 163 \\
\hline 9 & [69] & $\begin{array}{c}\text { EMS, EV, Charg- } \\
\text { ing/Discharging, } \\
\text { Photovoltaic System }\end{array}$ & Article & ITCED & IEEE & 2013 & $\begin{array}{l}\text { South } \\
\text { Korea }\end{array}$ & 131 \\
\hline 10 & [70] & $\begin{array}{l}\text { Eco-driving, EV, } \\
\text { Optimal control }\end{array}$ & Article & COEPE & $\begin{array}{l}\text { Elsevier } \\
\text { Ltd }\end{array}$ & 2014 & France & 126 \\
\hline 11 & [71] & $\begin{array}{l}\text { Asynchronous } \\
\text { machine, dc-link } \\
\text { voltage control, } \\
\text { converter, EM, FC, } \\
\text { HEV, LIB, SC }\end{array}$ & Article & ITVTA & IEEE & 2012 & France & 105 \\
\hline 12 & [72] & $\begin{array}{l}\text { DP, EMS, Global } \\
\text { optimization, } \\
\text { Modeling, PHEV }\end{array}$ & Article & Energies & MDPI AG & 2015 & China & 94 \\
\hline 13 & [61] & $\begin{array}{l}\text { BMS, EV, Charge } \\
\text { Equalization } \\
\text { Controller, Drive } \\
\text { Train Architecture }\end{array}$ & Review & RSERF & $\begin{array}{l}\text { Elsevier } \\
\text { Ltd }\end{array}$ & 2017 & Malaysia & 85 \\
\hline 14 & [73] & $\begin{array}{l}\text { EV, HESS, LIB, } \\
\text { Integrated } \\
\text { optimization, } \\
\text { Operation cost }\end{array}$ & Article & ENEYD & $\begin{array}{l}\text { Elsevier } \\
\text { Ltd }\end{array}$ & 2018 & China & 82 \\
\hline 15 & [74] & $\begin{array}{l}\text { BMS, ES, EV, } \\
\text { LIB, SOC }\end{array}$ & Review & Energies & MDPI AG & 2019 & $\begin{array}{l}\text { South } \\
\text { Korea }\end{array}$ & 80 \\
\hline 16 & [75] & $\begin{array}{c}\text { Brushless DC motor } \\
\text { drive, EV, ES, } \\
\text { FC, EMS }\end{array}$ & Review & RSERF & $\begin{array}{l}\text { Elsevier } \\
\text { Ltd }\end{array}$ & 2017 & $\begin{array}{l}\text { United } \\
\text { States }\end{array}$ & 80 \\
\hline 17 & [76] & $\begin{array}{c}\text { Fuel consumption, } \\
\text { PHEV, Quadratic } \\
\text { programming, } \\
\text { Simulated } \\
\text { annealing, SOH }\end{array}$ & Article & APEND & $\begin{array}{l}\text { Elsevier } \\
\text { Ltd }\end{array}$ & 2015 & $\begin{array}{l}\text { United } \\
\text { States }\end{array}$ & 80 \\
\hline 18 & [77] & $\begin{array}{l}\text { Electricity retailer } \\
\text { and smart grid, HSS, } \\
\text { PEV, Selling price } \\
\text { determination }\end{array}$ & Article & ECMAD & $\begin{array}{l}\text { Elsevier } \\
\text { Ltd }\end{array}$ & 2017 & Iran & 77 \\
\hline 19 & [78] & $\begin{array}{l}\text { Driving pattern, } \\
\text { EMS, OC, PHEV }\end{array}$ & Article & IETTE & IEEE & 2014 & $\begin{array}{l}\text { United } \\
\text { States }\end{array}$ & 72 \\
\hline 20 & [79] & $\begin{array}{l}\text { Energy saving, } \\
\text { Environmental } \\
\text { sustainability, } \\
\text { Metro-transit system, } \\
\text { PEV, Regenerative } \\
\text { braking }\end{array}$ & Article & EPSRD & $\begin{array}{l}\text { Elsevier } \\
\text { Ltd }\end{array}$ & 2011 & Italy & 72 \\
\hline
\end{tabular}


Table 2. Cont.

\begin{tabular}{|c|c|c|c|c|c|c|c|c|}
\hline Rank & Ref. no. & Keywords & $\begin{array}{l}\text { Type of } \\
\text { Article }\end{array}$ & $\begin{array}{l}\text { Abbreviated } \\
\text { Journal Name }\end{array}$ & $\begin{array}{l}\text { Publisher } \\
\text { Name }\end{array}$ & Year & Country & Citation \\
\hline 21 & [80] & $\begin{array}{l}\text { EM, fuel economy } \\
\text { benefits, heavy duty } \\
\text { diesel engines; HEV, } \\
\text { online optimization }\end{array}$ & Article & IETTE & IEEE & 2015 & $\begin{array}{l}\text { United } \\
\text { Kingdom }\end{array}$ & 66 \\
\hline 22 & [81] & $\begin{array}{c}\text { EV, LIB, MPC, PAC, } \\
\text { Remaining discharge } \\
\text { energy }\end{array}$ & Article & APEND & $\begin{array}{l}\text { Elsevier } \\
\text { Ltd }\end{array}$ & 2015 & China & 64 \\
\hline 23 & [82] & $\begin{array}{l}\text { Chevrolet Volt, NN, } \\
\text { genetic algorithm, } \\
\text { HEM }\end{array}$ & Article & ITVTA & IEEE & 2019 & China & 61 \\
\hline 24 & [83] & $\begin{array}{l}\text { EV, EV tools, Grid } \\
\text { tools, Smart grid; } \\
\text { V2G tools, VT }\end{array}$ & Review & APEND & $\begin{array}{l}\text { Elsevier } \\
\text { Ltd }\end{array}$ & 2016 & Australia & 61 \\
\hline 25 & [84] & $\begin{array}{c}\text { Battery lifetime, EV, } \\
\text { EMS, HESS, } \\
\text { Pontryagin's } \\
\text { minimum principle }\end{array}$ & Article & TSTE & IEEE & 2018 & China & 57 \\
\hline 26 & [85] & $\begin{array}{l}\text { Batteries, EMS, SC, } \\
\text { fully active parallel } \\
\text { topology, EV }\end{array}$ & Article & ITVTA & IEEE & 2017 & Canada & 53 \\
\hline 27 & [86] & $\begin{array}{l}\text { Basic operation } \\
\text { mode, EMS, } \\
\text { Modeling, PHEV }\end{array}$ & Article & Energies & MDPI AG & 2013 & China & 53 \\
\hline 28 & [87] & $\begin{array}{c}\text { Autonomous EV, } \\
\text { EM, Cyber-physical } \\
\text { systems, } \\
\text { Event-based control, } \\
\text { Wireless sensor } \\
\text { networks }\end{array}$ & Article & CMPJA & $\begin{array}{l}\text { Oxford } \\
\text { University } \\
\text { Press }\end{array}$ & 2013 & China & 50 \\
\hline 29 & [88] & $\begin{array}{c}\text { Battery, EM, } \\
\text { Flatness, FC, Fuzzy } \\
\text { logic, HV, SC }\end{array}$ & Article & ECMAD & $\begin{array}{l}\text { Elsevier } \\
\text { Ltd }\end{array}$ & 2019 & Tunisia & 39 \\
\hline 30 & [89] & $\begin{array}{l}\text { EMS, HEV, } \\
\text { Q-learning, } \\
\text { Reinforcement } \\
\text { learning }\end{array}$ & Article & APEND & $\begin{array}{l}\text { Elsevier } \\
\text { Ltd }\end{array}$ & 2020 & $\begin{array}{l}\text { United } \\
\text { States }\end{array}$ & 38 \\
\hline 31 & [90] & $\begin{array}{c}\text { battery life, EV, } \\
\text { EM, HESS }\end{array}$ & Article & ITPEE & IEEE & 2020 & China & 38 \\
\hline 32 & [91] & $\begin{array}{l}\text { BMS, HEV, SOC, } \\
\text { global positioning } \\
\text { system, Petri net, } \\
\text { rule-based strategy }\end{array}$ & Article & TASE & IEEE & 2017 & Egypt & 38 \\
\hline 33 & [92] & $\begin{array}{l}\text { Battery, EM, PHEV, } \\
\text { Component sizing, } \\
\text { Optimization }\end{array}$ & Article & Energies & MDPI AG & 2012 & $\begin{array}{l}\text { United } \\
\text { Kingdom }\end{array}$ & 37 \\
\hline 34 & [93] & $\begin{array}{l}\text { EV, ES, LIB, } \\
\text { SOC, SOH }\end{array}$ & Review & JEECS & ASME & 2019 & India & 35 \\
\hline 35 & [94] & $\begin{array}{l}\text { Deep reinforcement } \\
\text { learning, DP, EM, } \\
\text { MPC, Generalization }\end{array}$ & Article & ITVTA & IEEE & 2019 & China & 34 \\
\hline
\end{tabular}


Table 2. Cont.

\begin{tabular}{|c|c|c|c|c|c|c|c|c|}
\hline Rank & Ref. no. & Keywords & $\begin{array}{l}\text { Type of } \\
\text { Article }\end{array}$ & $\begin{array}{l}\text { Abbreviated } \\
\text { Journal Name }\end{array}$ & $\begin{array}{l}\text { Publisher } \\
\text { Name }\end{array}$ & Year & Country & Citation \\
\hline 36 & [95] & $\begin{array}{c}\text { Driving cycle } \\
\text { identification, EV, } \\
\text { EMS, Haar wavelet } \\
\text { transform }\end{array}$ & Article & Energies & MDPI AG & 2016 & China & 32 \\
\hline 37 & [96] & $\begin{array}{l}\text { EV, ES, fuzzy logic } \\
\text { control, genetic } \\
\text { algorithm, } \\
\text { optimization }\end{array}$ & Article & IJERD & $\begin{array}{l}\text { John Wiley } \\
\text { \& Sons Ltd }\end{array}$ & 2018 & Brazil & 30 \\
\hline 38 & [97] & $\begin{array}{c}\text { EV, EMS, FC, SC, } \\
\text { Grey wolf optimizer }\end{array}$ & Article & IJHED & $\begin{array}{l}\text { Elsevier } \\
\text { Ltd }\end{array}$ & 2019 & Algeria & 25 \\
\hline 39 & [98] & $\begin{array}{c}\text { EMS, FC, } \\
\text { Multi-objective } \\
\text { optimization, PHEV, } \\
\text { Velocity forecasting }\end{array}$ & Article & JPSOD & $\begin{array}{l}\text { Elsevier } \\
\text { B.V. }\end{array}$ & 2020 & France & 24 \\
\hline 40 & [99] & $\begin{array}{c}\text { DC-DC converter, } \\
\text { DTC-SVM, EV, } \\
\text { FC, PM }\end{array}$ & Article & JPSOD & $\begin{array}{l}\text { Elsevier } \\
\text { B.V. }\end{array}$ & 2020 & Algeria & 23 \\
\hline 41 & [100] & $\begin{array}{c}\text { EMS, HEV, } \\
\text { Markov chain, } \\
\text { Operation-mode } \\
\text { prediction }\end{array}$ & Article & JCROE & $\begin{array}{l}\text { Elsevier } \\
\text { Ltd }\end{array}$ & 2018 & China & 23 \\
\hline 42 & [101] & $\begin{array}{c}\text { Aircraft engine, EM, } \\
\text { HEV, Propulsion, } \\
\text { Vehicle sizing }\end{array}$ & Review & AATEE & $\begin{array}{l}\text { Emerald } \\
\text { Group } \\
\text { Holdings } \\
\text { Ltd. }\end{array}$ & 2014 & $\begin{array}{l}\text { United } \\
\text { States }\end{array}$ & 23 \\
\hline 43 & [102] & $\begin{array}{c}\text { HESS, EV, } \\
\text { Perturbation } \\
\text { observer, Robust } \\
\text { fractional-order } \\
\text { sliding-mode control }\end{array}$ & Article & JPSOD & $\begin{array}{l}\text { Elsevier } \\
\text { B.V. }\end{array}$ & 2020 & China & 21 \\
\hline 44 & [103] & $\begin{array}{l}\text { ECMS, EM, HEV, } \\
\text { OC, Pontryagin's } \\
\text { minimum principle }\end{array}$ & Article & APEND & $\begin{array}{l}\text { Elsevier } \\
\text { Ltd }\end{array}$ & 2017 & $\begin{array}{l}\text { United } \\
\text { States }\end{array}$ & 21 \\
\hline 45 & [104] & $\begin{array}{l}\text { Engine on/off } \\
\text { control, Estimation } \\
\text { distribution } \\
\text { algorithm, } \\
\text { Pontryagin's } \\
\text { minimum principle }\end{array}$ & Article & ENEYD & $\begin{array}{l}\text { Elsevier } \\
\text { Ltd }\end{array}$ & 2018 & China & 18 \\
\hline 46 & [105] & $\begin{array}{l}\text { EV, EM, OC, gain } \\
\text { scheduling, } \\
\text { linearization } \\
\text { techniques, real-time } \\
\text { simulation }\end{array}$ & Article & IETTE & IEEE & 2015 & France & 18 \\
\hline 47 & [106] & $\begin{array}{c}\text { EV, EMS, FC, SC, } \\
\text { permanent-magnet } \\
\text { synchronous motor }\end{array}$ & Article & ETEP & $\begin{array}{l}\text { John Wiley } \\
\text { \& Sons Ltd }\end{array}$ & 2017 & Algeria & 17 \\
\hline 48 & [107] & $\begin{array}{c}\text { ANN, forecasting, } \\
\text { Battery degradation } \\
\text { cost model, ES, EV, } \\
\text { Stochastic } \\
\text { programming }\end{array}$ & Article & SETA & $\begin{array}{l}\text { Elsevier } \\
\text { Ltd }\end{array}$ & 2020 & Iran & 16 \\
\hline
\end{tabular}


Table 2. Cont.

\begin{tabular}{|c|c|c|c|c|c|c|c|c|}
\hline Rank & Ref. no. & Keywords & $\begin{array}{l}\text { Type of } \\
\text { Article }\end{array}$ & $\begin{array}{c}\text { Abbreviated } \\
\text { Journal Name }\end{array}$ & $\begin{array}{c}\text { Publisher } \\
\text { Name }\end{array}$ & Year & Country & Citation \\
\hline 49 & [108] & $\begin{array}{l}\text { Adaptive equivalent } \\
\text { consumption } \\
\text { minimization } \\
\text { strategy, MPC, } \\
\text { PHEV }\end{array}$ & Article & ENEYD & $\begin{array}{l}\text { Elsevier } \\
\text { Ltd }\end{array}$ & 2020 & China & 14 \\
\hline 50 & [109] & $\begin{array}{l}\text { Adaptive controller, } \\
\text { Battery, EV, EMS, } \\
\text { Semi-active hybrid } \\
\text { energy storage } \\
\text { system, SC }\end{array}$ & Article & Energies & MDPI AG & 2019 & $\begin{array}{l}\text { South } \\
\text { Korea }\end{array}$ & 14 \\
\hline 51 & [110] & $\begin{array}{l}\text { Continuously } \\
\text { variable } \\
\text { transmission, EV, } \\
\text { HESS, SC }\end{array}$ & Article & ENEYD & $\begin{array}{l}\text { Elsevier } \\
\text { Ltd }\end{array}$ & 2019 & China & 14 \\
\hline 52 & [111] & $\begin{array}{c}\text { Energy optimization, } \\
\text { PHEV, RL, PM, } \\
\text { Q-learning }\end{array}$ & Article & TNNLS & IEEE & 2020 & $\begin{array}{l}\text { United } \\
\text { States }\end{array}$ & 13 \\
\hline 53 & [112] & $\begin{array}{c}\text { Automotive } \\
\text { applications, OC, } \\
\text { internal combustion } \\
\text { engines, nonlinear } \\
\text { control systems }\end{array}$ & Article & ITVTA & IEEE & 2018 & Spain & 13 \\
\hline 54 & [113] & $\begin{array}{c}\text { Dynamic } \\
\text { programming, MPC, } \\
\text { PEV, NN, } \\
\text { Pontryagin's } \\
\text { minimum principle }\end{array}$ & Article & ENEYD & $\begin{array}{l}\text { Elsevier } \\
\text { Ltd }\end{array}$ & 2020 & China & 12 \\
\hline 55 & [114] & $\begin{array}{l}\text { HEV, Hybrid sliding } \\
\text { mode controller, } \\
\text { Invasive weed } \\
\text { optimization }\end{array}$ & Article & EST & $\begin{array}{l}\text { Elsevier } \\
\text { Ltd }\end{array}$ & 2018 & Iran & 11 \\
\hline 56 & [115] & $\begin{array}{c}\text { Demand side } \\
\text { management, } \\
\text { Energy, EM, HEMS, } \\
\text { PEV, V2G }\end{array}$ & Article & Energies & MDPI AG & 2019 & Canada & 10 \\
\hline 57 & [116] & $\begin{array}{l}\text { fuzzy logic control, } \\
\text { HESS, EMS, PHEV, } \\
\text { SC, wavelet } \\
\text { transform }\end{array}$ & Article & IEEE Access & IEEE & 2018 & China & 10 \\
\hline 58 & [117] & $\begin{array}{c}\text { Dynamic } \\
\text { programming, EV, } \\
\text { EM, OC, Stochastic } \\
\text { systems }\end{array}$ & Article & IJAP & $\begin{array}{l}\text { SAE Inter- } \\
\text { national }\end{array}$ & 2013 & Germany & 10 \\
\hline 59 & [118] & $\begin{array}{c}\text { EM, HESS, PEV, } \\
\text { Temperature } \\
\text { uncertainty, Wavelet } \\
\text { transform }\end{array}$ & Article & APEND & $\begin{array}{l}\text { Elsevier } \\
\text { Ltd }\end{array}$ & 2019 & Australia & 9 \\
\hline 60 & [119] & $\begin{array}{c}\text { Diesel engine } \\
\text { modelling, EM, FC, } \\
\text { HEV, Multivariable } \\
\text { control systems, } \\
\text { Robust feedback } \\
\text { control }\end{array}$ & Article & IJVDD & $\begin{array}{l}\text { Inderscience } \\
\text { Publishers }\end{array}$ & 2012 & $\begin{array}{l}\text { United } \\
\text { Kingdom }\end{array}$ & 9 \\
\hline
\end{tabular}


Table 2. Cont.

\begin{tabular}{|c|c|c|c|c|c|c|c|c|}
\hline Rank & Ref. no. & Keywords & $\begin{array}{l}\text { Type of } \\
\text { Article }\end{array}$ & $\begin{array}{l}\text { Abbreviated } \\
\text { Journal Name }\end{array}$ & $\begin{array}{c}\text { Publisher } \\
\text { Name }\end{array}$ & Year & Country & Citation \\
\hline 61 & [120] & $\begin{array}{l}\text { EMS, RL, Markov } \\
\text { chain, Stochastic } \\
\text { model prediction } \\
\text { control, Velocity } \\
\text { prediction }\end{array}$ & Article & ENEYD & $\begin{array}{l}\text { Elsevier } \\
\text { Ltd }\end{array}$ & 2020 & China & 8 \\
\hline 62 & [121] & $\begin{array}{l}\text { Direct refrigerant } \\
\text { cooling, EV, } \\
\text { LIB, EMS }\end{array}$ & Article & ESD & $\begin{array}{c}\text { Elsevier } \\
\text { B.V. }\end{array}$ & 2020 & China & 8 \\
\hline 63 & [122] & $\begin{array}{l}\text { fuel consumption, } \\
\text { Grey wolf optimizer, } \\
\text { HEV, rules-based } \\
\text { energy management }\end{array}$ & Article & TICOD & $\begin{array}{l}\text { SAGE Pub- } \\
\text { lications } \\
\text { Ltd }\end{array}$ & 2020 & Tunisia & 8 \\
\hline 64 & [123] & $\begin{array}{l}\text { EV, EMS, FC, } \\
\text { HEV, Energetic } \\
\text { macroscopic } \\
\text { representation }\end{array}$ & Article & MCSID & $\begin{array}{c}\text { Elsevier } \\
\text { B.V. }\end{array}$ & 2020 & France & 8 \\
\hline 65 & [124] & $\begin{array}{l}\text { Intelligent energy } \\
\text { management, } \\
\text { Multi-agent; } \\
\text { Proton Membrane } \\
\text { Exchange fuel cell, } \\
\text { Real-time, SC } \\
\end{array}$ & Article & Energies & MDPI AG & 2019 & Tunisia & 8 \\
\hline 66 & [125] & $\begin{array}{l}\text { EV, Loop Heat Pipe, } \\
\text { Lumped parameter, } \\
\text { Thermal } \\
\text { management }\end{array}$ & Article & ATENF & $\begin{array}{l}\text { Elsevier } \\
\text { Ltd }\end{array}$ & 2018 & $\begin{array}{l}\text { United } \\
\text { Kingdom }\end{array}$ & 8 \\
\hline 67 & [126] & $\begin{array}{c}\text { Connected and } \\
\text { automated vehicles, } \\
\text { hierarchical model } \\
\text { predictive control, } \\
\text { thermal } \\
\text { management }\end{array}$ & Article & IETTE & IEEE & 2021 & $\begin{array}{l}\text { United } \\
\text { States }\end{array}$ & 7 \\
\hline 68 & [127] & $\begin{array}{c}\text { Battery degradation, } \\
\text { EV, EM, HESS, } \\
\text { Sizing }\end{array}$ & Article & ENEYD & $\begin{array}{l}\text { Elsevier } \\
\text { Ltd }\end{array}$ & 2020 & $\begin{array}{l}\text { United } \\
\text { Kingdom }\end{array}$ & 7 \\
\hline 69 & [128] & $\begin{array}{l}\text { EMS, FC, HEV, } \\
\text { Hierarchical } \\
\text { clustering, } \\
\text { Rule learning }\end{array}$ & Article & JCROE & $\begin{array}{l}\text { Elsevier } \\
\text { Ltd }\end{array}$ & 2020 & China & 7 \\
\hline 70 & [129] & $\begin{array}{l}\text { Back propagation } \\
\text { NN, EMS, HEV, } \\
\text { Compound } \\
\text { structured } \\
\text { permanent-magnet } \\
\text { motor }\end{array}$ & Article & Energies & MDPI AG & 2018 & China & 7 \\
\hline 71 & [130] & $\begin{array}{c}\text { Dual droop control, } \\
\text { EV, HESS, } \\
\text { Frequency diving } \\
\text { coordinated control }\end{array}$ & Article & JMPSCE & Springer & 2015 & China & 7 \\
\hline 72 & [131] & $\begin{array}{c}\text { Construction vehicle, } \\
\text { EM, FC, MPC, } \\
\text { NN, Wavelet }\end{array}$ & Article & ENEYD & $\begin{array}{l}\text { Elsevier } \\
\text { Ltd }\end{array}$ & 2020 & China & 6 \\
\hline
\end{tabular}


Table 2. Cont

\begin{tabular}{|c|c|c|c|c|c|c|c|c|}
\hline Rank & Ref. no. & Keywords & $\begin{array}{l}\text { Type of } \\
\text { Article }\end{array}$ & $\begin{array}{l}\text { Abbreviated } \\
\text { Journal Name }\end{array}$ & $\begin{array}{l}\text { Publisher } \\
\text { Name }\end{array}$ & Year & Country & Citation \\
\hline 73 & [132] & $\begin{array}{l}\text { Distributed energy } \\
\text { management, V2G, } \\
\text { greedy-based } \\
\text { algorithm, mixed } \\
\text { integer non-linear } \\
\text { programming }\end{array}$ & Article & IEEE Access & IEEE & 2020 & $\begin{array}{l}\text { United } \\
\text { Kingdom }\end{array}$ & 5 \\
\hline 74 & [133] & $\begin{array}{c}\text { Algorithm, } \\
\text { Classification, EMS, } \\
\text { HEV, Optimization }\end{array}$ & Review & Energies & MDPI AG & 2020 & China & 4 \\
\hline 75 & [134] & $\begin{array}{l}\text { Batteries, EMS, FC, } \\
\text { EV, Fuzzy inference } \\
\text { system, Hull moving } \\
\text { average }\end{array}$ & Article & Energies & MDPI AG & 2019 & China & 4 \\
\hline 76 & [135] & $\begin{array}{c}\text { Equivalent } \\
\text { Consumption } \\
\text { Minimization } \\
\text { Strategy, equivalent } \\
\text { factor, fuzzy logic }\end{array}$ & Article & JIFS & IOS Press & 2017 & China & 4 \\
\hline 77 & [136] & $\begin{array}{l}\text { Advanced model, } \\
\text { battery lifetime, EV, } \\
\text { EMS, HESS, LIB, SC }\end{array}$ & Article & ITIED & IEEE & 2021 & France & 3 \\
\hline 78 & [137] & $\begin{array}{l}\text { BMS, EV, LIB, } \\
\text { Cost estimation, } \\
\text { Fiber optic sensor }\end{array}$ & Review & Sensors & MDPI AG & 2021 & $\begin{array}{l}\text { United } \\
\text { States }\end{array}$ & 3 \\
\hline 79 & [138] & $\begin{array}{l}\text { Charging (batteries), } \\
\text { EV, EE, EM, EPTN }\end{array}$ & Article & RPG & $\begin{array}{l}\text { John Wiley } \\
\text { \& Sons Inc }\end{array}$ & 2020 & Denmark & 3 \\
\hline 80 & [139] & $\begin{array}{l}\text { Bidirectional power } \\
\text { flow, DC-DC } \\
\text { converters, EV, SC }\end{array}$ & Article & EENGF & Springer & 2020 & Brazil & 3 \\
\hline 81 & [140] & $\begin{array}{l}\text { Battery, EV, EMS, SC, } \\
\text { Jaya algorithm }\end{array}$ & Article & IJERD & $\begin{array}{l}\text { John Wiley } \\
\text { \& Sons Inc }\end{array}$ & 2020 & Turkey & 3 \\
\hline 82 & [141] & $\begin{array}{c}\text { SOC, DC, EM, FC, } \\
\text { HEV, Pattern } \\
\text { recognition, } \\
\text { Supervisory control }\end{array}$ & Article & IJEHV & $\begin{array}{l}\text { Inderscience } \\
\text { Publishers }\end{array}$ & 2010 & Iran & 3 \\
\hline 83 & [142] & $\begin{array}{l}\text { Fuzzy based EM, } \\
\text { HESS, FC, Super } \\
\text { twisting sliding } \\
\text { mode control }\end{array}$ & Article & EST & $\begin{array}{l}\text { Elsevier } \\
\text { Ltd }\end{array}$ & 2021 & Pakistan & 2 \\
\hline 84 & [143] & $\begin{array}{l}\text { Bidirectional DC-DC } \\
\text { converter, EV, FC, } \\
\text { Real time digital } \\
\text { simulator }\end{array}$ & Article & JPE & $\begin{array}{c}\text { Korean } \\
\text { Institute of } \\
\text { Power } \\
\text { Electronics }\end{array}$ & 2011 & $\begin{array}{l}\text { United } \\
\text { States }\end{array}$ & 2 \\
\hline 85 & [144] & $\begin{array}{c}\text { EV, EE, NN, Fuzzy } \\
\text { logic, Intelligent } \\
\text { controllers, } \\
\text { Regenerative } \\
\text { braking }\end{array}$ & Review & Energies & MDPI AG & 2021 & Estonia & 1 \\
\hline
\end{tabular}


Table 2. Cont.

\begin{tabular}{|c|c|c|c|c|c|c|c|c|}
\hline Rank & Ref. no. & Keywords & $\begin{array}{l}\text { Type of } \\
\text { Article }\end{array}$ & $\begin{array}{c}\text { Abbreviated } \\
\text { Journal Name }\end{array}$ & $\begin{array}{c}\text { Publisher } \\
\text { Name }\end{array}$ & Year & Country & Citation \\
\hline 86 & [145] & $\begin{array}{c}\text { ES, EV, Isolated } \\
\text { power grids, } \\
\text { Transport } \\
\text { decarbonization, } \\
\text { V2G }\end{array}$ & Article & Energies & MDPI AG & 2021 & Portugal & 1 \\
\hline 87 & [146] & $\begin{array}{l}\text { battery swapping } \\
\text { station, EV, V2G, } \\
\text { stochastic model } \\
\text { predictive control }\end{array}$ & Article & IJERD & $\begin{array}{l}\text { John Wiley } \\
\& \text { Sons Ltd }\end{array}$ & 2021 & China & 1 \\
\hline 88 & [147] & $\begin{array}{l}\text { EV, EM, Energy } \\
\text { consumption, } \\
\text { Supply chain, } \\
\text { Vehicle routing } \\
\text { problem }\end{array}$ & Article & Energies & MDPI AG & 2021 & $\begin{array}{l}\text { United } \\
\text { States }\end{array}$ & 1 \\
\hline 89 & [148] & $\begin{array}{c}\text { Commercial } \\
\text { building, EV, retired } \\
\text { electric vehicle } \\
\text { battery, Risk } \\
\text { management } \\
\text { strategy }\end{array}$ & Article & ECMAD & $\begin{array}{l}\text { Elsevier } \\
\text { Ltd }\end{array}$ & 2021 & China & 1 \\
\hline 90 & [149] & $\begin{array}{c}\text { Auxiliary power } \\
\text { unit, Charging } \\
\text { strategy, Cost } \\
\text { analysis, EM, HESS }\end{array}$ & Review & RSERF & $\begin{array}{l}\text { Elsevier } \\
\text { Ltd }\end{array}$ & 2021 & Australia & 0 \\
\hline 91 & [150] & $\begin{array}{c}\text { Coolant, direct } \\
\text { cooling system, EV, } \\
\text { LIB, two-phase flow }\end{array}$ & Review & IJERD & $\begin{array}{l}\text { John Wiley } \\
\text { \& Sons Ltd }\end{array}$ & 2021 & China & 0 \\
\hline 92 & [151] & $\begin{array}{l}\text { Cost optimization, } \\
\text { EV, EM, HESS, NN, } \\
\text { Variable perception } \\
\text { horizon }\end{array}$ & Article & APEND & $\begin{array}{l}\text { Elsevier } \\
\text { Ltd }\end{array}$ & 2021 & $\begin{array}{l}\text { United } \\
\text { Kingdom }\end{array}$ & 0 \\
\hline 93 & [152] & $\begin{array}{c}\text { Deep Q learning, } \\
\text { HEV, MPC, } \\
\text { Prioritized replay }\end{array}$ & Article & ENEYD & $\begin{array}{l}\text { Elsevier } \\
\text { Ltd }\end{array}$ & 2021 & China & 0 \\
\hline 94 & [153] & $\begin{array}{c}\text { Dynamic } \\
\text { programming, } \\
\text { Electrified } \\
\text { powertrain, EMS, } \\
\text { OC, HEV }\end{array}$ & Article & Energies & MDPI AG & 2021 & Italy & 0 \\
\hline 95 & [154] & $\begin{array}{c}\text { Battery, } \\
\text { Gain scheduled, } \\
\text { Linear parameter } \\
\text { varying, SC }\end{array}$ & Article & ITCNE & IEEE & 2021 & France & 0 \\
\hline 96 & [155] & $\begin{array}{l}\text { Energy harvesting, } \\
\text { EM, HEV, SC }\end{array}$ & Article & Energies & MDPI AG & 2021 & Greece & 0 \\
\hline 97 & [156] & $\begin{array}{l}\text { hybrid sources, } \\
\text { LIB, SC }\end{array}$ & Review & IJERD & $\begin{array}{l}\text { John Wiley } \\
\& \text { Sons Ltd }\end{array}$ & 2021 & China & 0 \\
\hline 98 & [157] & $\begin{array}{c}\text { EMS, MPC, } \\
\text { integrated power } \\
\text { system, load power } \\
\text { prediction }\end{array}$ & Article & IEEE Access & IEEE & 2021 & China & 0 \\
\hline
\end{tabular}


Table 2. Cont.

\begin{tabular}{|c|c|c|c|c|c|c|c|c|}
\hline Rank & Ref. no. & Keywords & $\begin{array}{l}\text { Type of } \\
\text { Article }\end{array}$ & $\begin{array}{l}\text { Abbreviated } \\
\text { Journal Name }\end{array}$ & $\begin{array}{l}\text { Publisher } \\
\text { Name }\end{array}$ & Year & Country & Citation \\
\hline 99 & [158] & $\begin{array}{l}\text { Adaptive equivalent } \\
\text { consumption } \\
\text { minimum strategy, } \\
\text { equivalent factor, } \\
\text { PHEV }\end{array}$ & Article & IJERD & $\begin{array}{l}\text { John Wiley } \\
\text { \& Sons Ltd }\end{array}$ & 2021 & China & 0 \\
\hline 100 & [159] & $\begin{array}{l}\text { DC-DC converter; } \\
\text { EV; intelligent } \\
\text { controller; BSS; } \\
\text { modulation } \\
\text { techniques; } \\
\text { metaheuristic } \\
\text { optimization }\end{array}$ & Review & Electronics & MDPI AG & 2021 & Malaysia & 0 \\
\hline
\end{tabular}

$\mathrm{AB}=$ Automotive Batteries, ANN = Artificial Neural Network, BMS = Battery Management Systems, BP = Battery Pack, BSOC $=$ Battery State of Charge, BESS = Battery Energy Storage Systems, CA = Cost Analysis, CR = Cost Reduction, CS = Control System, DP = Dynamic Programming, EB = Electric Batteries, EE = Energy Efficiency, ED = Electric Discharges, EM = Energy Management, EMC = Electric Machine Control, EP = Energy Planning, EPTN = Electric Power Transmission Networks, EV = Electric Vehicle, EMS = Energy Management Systems, ES = Energy Storage, ESS = Energy Storage Systems, EU = Energy Utilization, FC = Fuel Cells, FE = Fuel Economy, FL = Fuzzy Logic, HESS = Hybrid Energy Storage Systems, HV = Hybrid Vehicles, HEV = Hybrid Electric Vehicles, LIB = Lithium-ion Batteries, MPC = Model Predictive Control, NN = Neural Networks, OCS = Optimal Control Systems, OC = Optimal Control, PCS $=$ Predictive Control Systems, PHEV = Plug-in Hybrid Electric Vehicles, PHV = Plug-in Hybrid Vehicles, PM = Power Management, REM = Real-time Energy Management, RL = Reinforcement Learning, SC = Solar Cells, SOC = State of Charge, $\mathrm{SM}=$ Storage Management, $\mathrm{SS}=\mathrm{Stoch}$ astic Systems, SB = Secondary Batteries, SC = Supercapacitor, TC = Temperature Control, VA = Vehicle Applications, V2G = Vehicle-to-grid.

\section{Analytical Discussion}

The analysis of the most relevant article in any specific field of study is critical for understanding and categorizing current research trends and providing an overall idea about the influential journals and publications. We aimed to provide transparent information about the most important fields of research manuscripts and recent research developments in EMSs for EV applications with this study.

\subsection{Citation Analysis of the Selected Most Relevant Manuscripts}

Table 2 shows the 100 most relevant articles in the field of EMSs for EV applications, as extracted from the Scopus database and analyzed to deliver further information for future researchers. It can be observed that Table 2 illustrates the number of citations for the 100 manuscripts, whose citation numbers range between 0 and 673; the first 6 manuscripts received more than 200 citations, while the first 11 manuscripts had more than 100 citations. Hannan et al. generated the manuscripts with the highest citations in 2017.

The most cited article in the field of EMSs for EV applications is "A review of lithiumion battery state of charge estimation and management system in EV applications: Challenges and recommendations" [62] produced by Hannan et al., which received 673 citations and was published in the journal "Renewable and Sustainable Energy Reviews" in 2017. This study estimates the lithium-ion battery state of charge (SOC) and examines its management system in the context of future EV applications. Moreover, the need for a lithium-ion battery management system (BMS) is discussed, ensuring a dependable and safe operation while also assessing the battery's state of charge (SOC). The SOC is a critical statistic, according to the study, since it indicates the remaining available energy in a battery, which gives an indication of charging/discharging strategies and protects the battery from overcharging/over-discharging. According to the citation, "Battery management system: An overview of its application in the smart grid and EVs" is a review paper by Rahimi-Eichi et al. that evaluates battery management issues based on smart grids and EV [63]. In 2013, the work was published in the journal "IEEE Industrial Electronics Magazine", and it received 487 citations. "Review of energy storage systems for EV applications: Issues and challenges" [6] was the third most cited manuscript published in the "Renewable and Sustainable Energy Reviews" journal in 2017. Hannan et al. authored the manuscript, which 
received 384 citations. This manuscript examines energy storage system (ESS) technologies in detail, including classifications, features, structures, power conversion, and assessment procedures, as well as their benefits and drawbacks in EV applications. Furthermore, this article examines several classes of ESS based on their energy forms, composition materials, and methodologies in terms of the average power delivery overcapacity and overall efficiency displayed within their life expectancies. Articles with an average citation per year (ACY) of 21.6 or higher are considered the most important in the EMS field. Those included in Table 2 provide a more profound knowledge of the topic.

\subsection{Allocation of the Selected 100 Manuscripts over the Year}

In Figure 3, the allocation of the 100 stated articles in EMSs for EV applications between 2010 and 2021 is illustrated. The numbers of papers published in the years 2010 and 2011 are 1 and 2, respectively. Based on Figure 3, the number of manuscripts published in 2020 was the highest, while it was the lowest in 2010; the figures are 22 and 1, respectively. With 12 manuscripts each, the number of papers generated in 2018 and 2019 is the same. Overall, the articles published from 2019 to 2021 indicate an increasing trend, but those-published from 2010 to 2016 show a fluctuating trend.

25

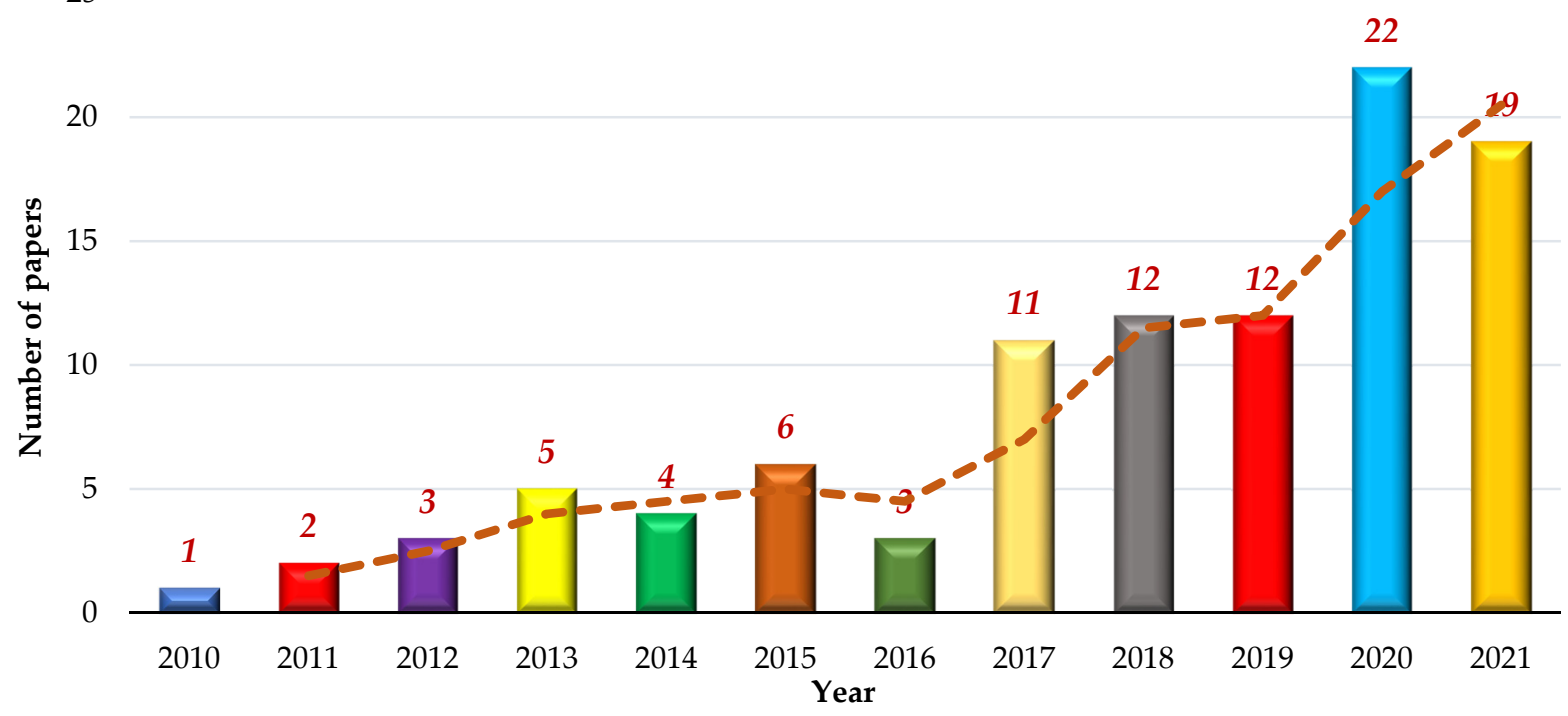

Figure 3. Distribution of elected manuscripts over the years 2010 to 2021.

\subsection{Co-Occurrence Keyword Analysis}

Table 2 delivers a broad notion of the selected research area, while Figure 4 shows co-occurrence keywords from the most relevant manuscripts picked from the selected database. Figure 4 demonstrates the internal network among all keywords, which is generated by using the VOSviewer software. The influence of the keywords controls the volume of the circle and label, while the connecting line among the keywords is revealed as a conjunctive connection. Different colors are used to describe different clusters depending on the area of expertise. The adaptive controller, battery, battery degradation cost model, battery management system, charge equalization controller, coolant, cost estimation, deep neural network, dual droop control, EV, energy storage, genetic algorithm, home energy management system, hydrogen fuel consumption, integrated energy management strategy, integrated optimization, isolated power grids, Jaya algorithm, optimization, parameter match, risk management strategy, sector-coupling, smart grid, state of charge, temperature monitoring, thermal management, thermal runaway, transport decarbonization, and variable perception horizon are in the red cluster, which illustrates the strong bond among them. The blue cluster represents different sorts of energy management strategies such 
as the adaptive controller, wavelet transform, hybrid energy storage system, vehicle routing problem, simulation, supercapacitor, charging strategy, auxiliary power unit, battery lifetime, energy harvesting, hybrid power systems, autonomous EV, event-based control, demand-side management, equivalent factor, fuzzy logic, propulsion, energy management, online optimization, and energy efficiency that are considered to smooth energy transition. It can be noticed that environmental sustainability, regenerative braking, intelligent controllers, polynomial control, speed control, current control method, fuel cell, dc/ac converter, dc-link voltage control, multi-objective optimization, asynchronous machine, dc/dc converter, neural network, Pontryagin's minimum principle, gain scheduling, realtime simulation, fuel economy, pattern recognition, driving cycle, and optimal control are directly connected to the energy controller, which is represented in the purple cluster. The optimizations are directly related to look-ahead control, dynamic programming, generalization, model predictive control, deep reinforcement learning, uncertain systems, multivariable control systems, robust feedback control, load power prediction, hybrid EV, fuel efficiency, deep q learning, global optimization, modeling, and basic operation mode, which are in the green cluster. Finally, plug-in hybrid EV, quadratic programming, state of health, fuel consumption, grey wolf optimizer, rules-based energy management, deep q-learning, reinforcement learning, the Markov chain, energy loss, the forgetting factor, power transition probability matrices, and Kullback-Leibler divergence are strongly linked to EV storage efficiency, which is represented by the yellow cluster.

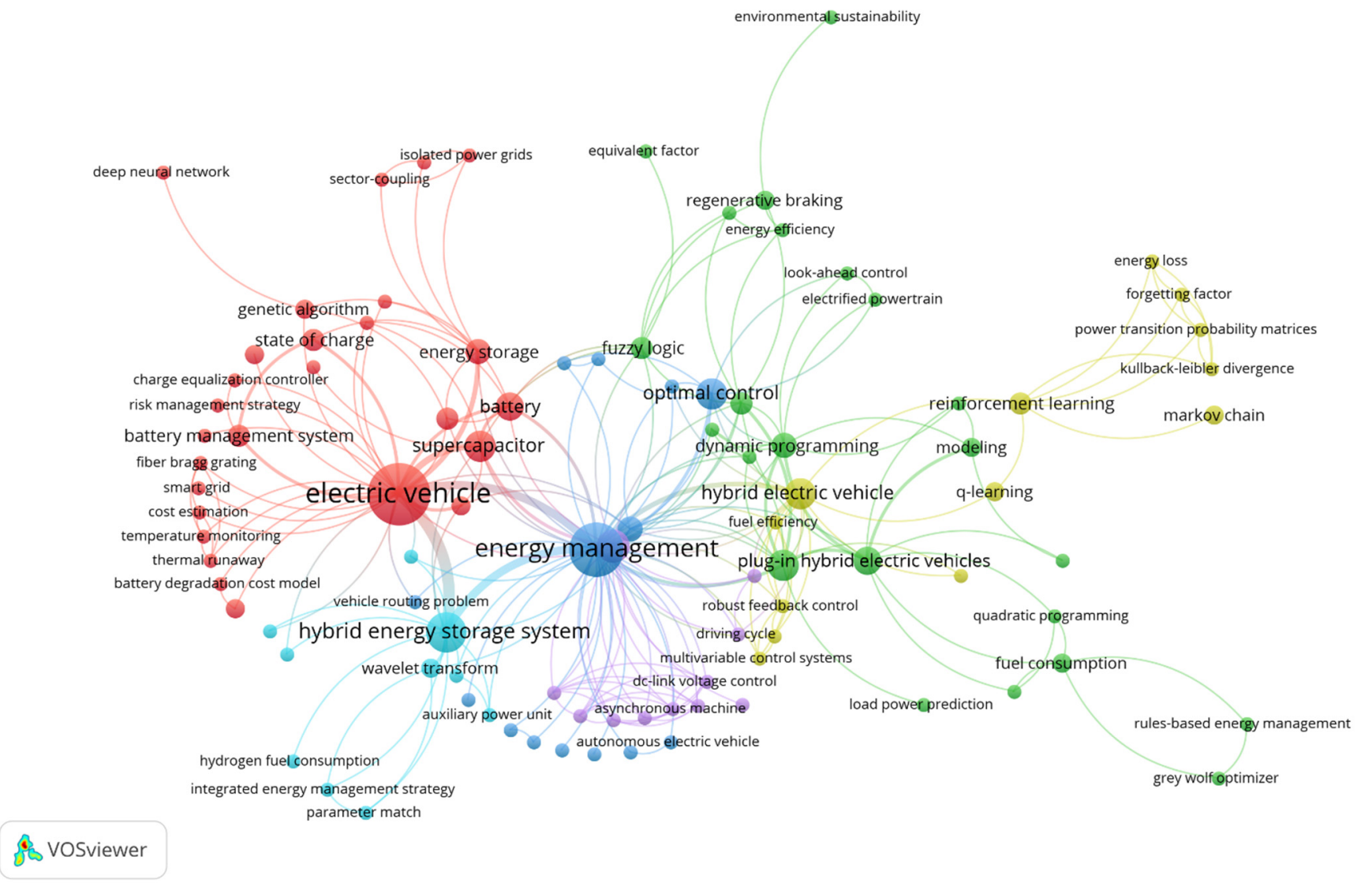

Figure 4. Co-occurrence keywords analysis by VOSviewer from the Scopus database.

Table 3 reveals the topmost 15 keywords from the chosen database used in multiple publications between 2010 and 2021. The current literature gaps can be discovered by analyzing the topmost keywords, and insight into the recent research field can be obtained. "Energy Management Systems", "Electric Vehicles", and "Secondary Batteries" are the three most prevalent terms in Table 2. The values for "Energy Management Systems" and 
"Electric Vehicles" are 42 and 35, respectively, while "Secondary Batteries" have a figure of 31. "Energy Management Systems", "Electric Vehicles", "Optimization", and "Controllers" were also the most popular terms in the recent two years, reflecting the growing interest in EMSs for EV applications. The total allocation of keywords and the graphical depiction of Table 3 are depicted in Figure 5. Based on the analysis of Table 3 and Figure 5, the following conclusions can be made:

- Scholars are now concerned about energy storage efficiency and minimizing carbon's impact on the climate while enhancing the system's efficiency.

- There has been a tremendous rise in EMS and EV application research.

Number of articles

Frequency

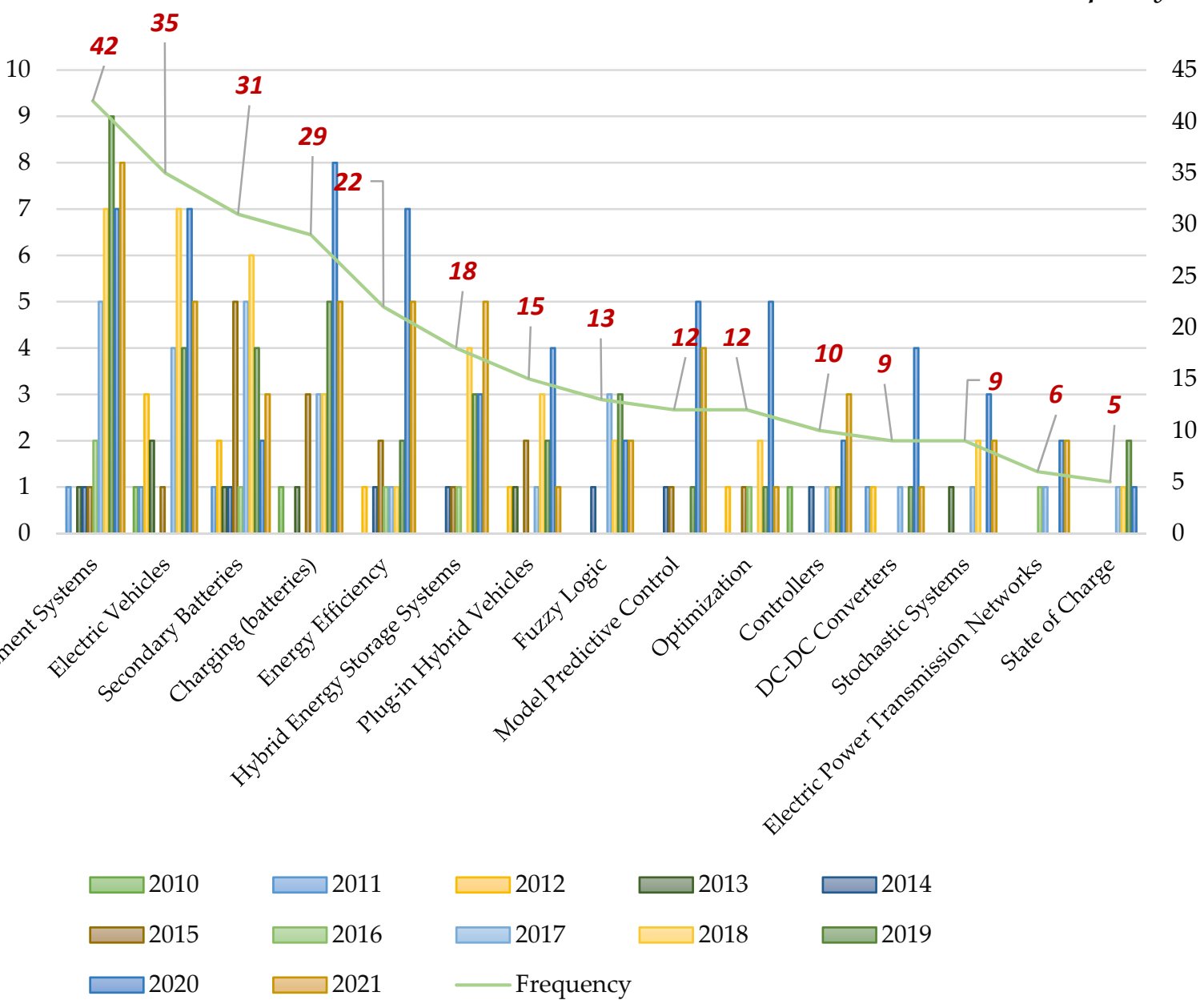

Figure 5. Distribution of topmost 15 keywords over the year 2010 to 2021.

According to Tables 2 and 3, it is clear that the scholars are currently interested in EMSs for EV application development, particularly in control and optimization technologies, topics that have received a lot of citations in the last 5 years and have a lot more ACY than the topic of general energy efficiency in storage systems. 
Table 3. Top-most 15 keywords from the selected 100 manuscripts between 2010 and 2021 .

\begin{tabular}{|c|c|c|c|c|c|c|c|c|c|c|c|c|c|}
\hline Top Keywords & 2010 & 2011 & 2012 & 2013 & 2014 & 2015 & 2016 & 2017 & 2018 & 2019 & 2020 & 2021 & Frequency \\
\hline $\begin{array}{c}\text { Energy Management } \\
\text { Systems }\end{array}$ & & [79] & & [69] & {$[66]$} & [105] & {$[68,95]$} & $\begin{array}{c}{[61,62,75,} \\
77,85]\end{array}$ & $\begin{array}{l}{[64,65,73,84} \\
100,114,125]\end{array}$ & $\begin{array}{c}{[82,88,94,97} \\
105,109,118 \\
124,134]\end{array}$ & $\begin{array}{c}{[89,107,123,132} \\
133,138,140]\end{array}$ & $\begin{array}{c}\text { [142,145,148,149, } \\
151,154-156]\end{array}$ & 42 \\
\hline Secondary Batteries & & [143] & {$[71,92]$} & [86] & {$[66]$} & $\begin{array}{l}{[76,80,81} \\
105,130] \\
\end{array}$ & [95] & $\begin{array}{c}{[61,62,85,} \\
91,103]\end{array}$ & $\begin{array}{c}{[64,73,84,104,} \\
116]\end{array}$ & $\begin{array}{c}{[88,109,118,} \\
134]\end{array}$ & {$[123,140]$} & {$[142,149,151]$} & 31 \\
\hline Charging (batteries) & [141] & & & [86] & & {$[80,81,130]$} & & {$[62,91,103]$} & {$[84,104,129]$} & $\begin{array}{c}{[74,88,93,109,} \\
115]\end{array}$ & $\begin{array}{c}{[98,99,107,111} \\
120,122,128,138]\end{array}$ & $\begin{array}{c}142,146,149,157, \\
158]\end{array}$ & 29 \\
\hline Energy Efficiency & & & [119] & & [66] & {$[80,81]$} & {$[68]$} & [91] & [64] & {$[88,110]$} & $\begin{array}{c}{[89,99,122,131,} \\
133,138-140]\end{array}$ & $\begin{array}{c}{[144,149,150,150} \\
158]\end{array}$ & 22 \\
\hline $\begin{array}{l}\text { Hybrid Energy } \\
\text { Storage Systems }\end{array}$ & & & & & {$[66]$} & [130] & {$[68]$} & & {$[64,73,84,116]$} & {$[109,110,118]$} & {$[90,99,102]$} & $\begin{array}{c}{[136,142,149,151} \\
156]\end{array}$ & 18 \\
\hline $\begin{array}{l}\text { Plug-in Hybrid } \\
\text { Vehicles }\end{array}$ & & & [92] & [86] & & {$[72,76]$} & & [135] & {$[64,104,116]$} & {$[82,118]$} & $\begin{array}{c}{[108,111,113,} \\
120]\end{array}$ & [158] & 15 \\
\hline Fuzzy Logic & & & & & [66] & & & {$[85,91,135]$} & {$[96,116]$} & {$[88,109,118]$} & {$[90,99]$} & {$[142,144]$} & 13 \\
\hline $\begin{array}{l}\text { Model Predictive } \\
\text { Control }\end{array}$ & & & & & [101] & [81] & & & & [94] & $\begin{array}{c}{[98,108,113,120} \\
131]\end{array}$ & {$[126,146,152,157]$} & 12 \\
\hline Optimization & & & [92] & & & [80] & {$[68]$} & & {$[73,96]$} & [118] & $\begin{array}{c}{[89,122,127,131} \\
133]\end{array}$ & [144] & 12 \\
\hline Controllers & [141] & & & & {$[66]$} & & & [61] & [114] & [109] & {$[102,120]$} & {$[142,144,154]$} & 10 \\
\hline DC-DC Converters & & [143] & [71] & & & & & [106] & & [118] & {$[90,102,123,139]$} & [154] & 9 \\
\hline Stochastic Systems & & & & [117] & & & & [77] & {$[100,114]$} & & {$[107,120,132]$} & {$[146,148]$} & 9 \\
\hline State of Charge & & & & & & & & [62] & [84] & {$[74,93]$} & [120] & [160-162] & 8 \\
\hline $\begin{array}{c}\text { Electric Power } \\
\text { Transmission } \\
\text { Networks }\end{array}$ & & & & & & & [83] & [77] & & & {$[132,138]$} & {$[145,146]$} & 6 \\
\hline
\end{tabular}




\subsection{Bibliometric Analysis of Average Citations per Year and Study Type}

Table 4 shows the top ten manuscripts with the greatest ACY in the last five years. Moreover, Table 4 also demonstrates the contributions, achievements, research gaps, and future directions in the top ten selected manuscripts. The most frequent research gaps include a lack of working capability in the real-world environment, a lack of accurate and robust SOC estimation under real-time EV drive cycles, short lifetime and limited load capacity, high cost, marginal safety, small voltage, and low energy density. In line with this, numerous key factors were identified including expenses, safety measures, sizing, power electronics interfaces, energy and power management, proper disposal, recycling, and material support. To overcome the above-mentioned knowledge gaps from different studies related to EMS in EVs, further investigation is required. The article by Hannan et al. has the highest ACY of 133.6, which is also ranked first in the total number of citations, followed by the second article by Rahimi-Eichi et al., which has an ACY of 73.8. As the research interests of scholars change over time, the ACY rank varies in relation to the overall citation rank.

Table 4. "Average citation per year (ACY)" of top 10 articles.

\begin{tabular}{|c|c|c|c|c|c|c|}
\hline Rank & Ref. & $\mathrm{ACY}$ & $\begin{array}{c}\text { Citation Rank } \\
\text { Based on Table } 2\end{array}$ & $\begin{array}{l}\text { Abbreviated } \\
\text { Keywords }\end{array}$ & Contributions & $\begin{array}{c}\text { Research Gaps/Future } \\
\text { Directions }\end{array}$ \\
\hline 1 & {$[62]$} & 133.6 & 1 & $\begin{array}{l}\text { BMS, EV, } \\
\text { LIB, SOC }\end{array}$ & $\begin{array}{l}\text { This research examines the } \\
\text { estimation of Li-ion battery } \\
\text { SOC and its EMS in the } \\
\text { context of future } \\
\text { EV applications. }\end{array}$ & $\begin{array}{l}\text { - Lack of working } \\
\text { capability in real } \\
\text { applications. } \\
\text { - SOC estimation is only } \\
\text { possible when the EVs } \\
\text { are not moving. }\end{array}$ \\
\hline 2 & [63] & 73.8 & 2 & $\begin{array}{c}\text { BMS, BT, } \\
\text { Charge/discharge, } \\
\text { EV, SOC, SOH }\end{array}$ & $\begin{array}{l}\text { This study evaluates the } \\
\text { performance of BMS } \\
\text { concerning reliability, } \\
\text { safety, and cost. }\end{array}$ & $\begin{array}{l}\text { Various batteries have } \\
\text { distinct constraints on the } \\
\text { charge receiving due to } \\
\text { their different chemistries } \\
\text { and architectures. }\end{array}$ \\
\hline 3 & [6] & 76.2 & 3 & $\begin{array}{c}\text { EV, ESS, } \\
\text { Hybridization, } \\
\text { Power electronics }\end{array}$ & $\begin{array}{l}\text { This research assesses the } \\
\text { different composition } \\
\text { materials and } \\
\text { methodologies of ESS } \\
\text { based on average power } \\
\text { delivery, capacity, and } \\
\text { efficiency within } \\
\text { their lifetime. }\end{array}$ & $\begin{array}{l}\text { Crucial factors such as } \\
\text { expenses, safety } \\
\text { measures, sizing, power } \\
\text { electronics interface, } \\
\text { energy and power } \\
\text { management, proper } \\
\text { disposal, recycling, and } \\
\text { material support are } \\
\text { not considered. }\end{array}$ \\
\hline 4 & {$[65]$} & 52.5 & 5 & $\begin{array}{l}\text { EV, EMS, } \\
\text { LIB, SOC }\end{array}$ & $\begin{array}{l}\text { The reinforcement learning } \\
\text { (RL)-based real-time } \\
\text { power-management } \\
\text { approach is used to } \\
\text { achieve the optimal power } \\
\text { distribution between the } \\
\text { battery and SC. }\end{array}$ & $\begin{array}{l}\text { As the outcomes of this } \\
\text { paper are satisfactory and } \\
\text { efficient, this technique } \\
\text { can be implemented for } \\
\text { practical purposes. }\end{array}$ \\
\hline 5 & [67] & 46.25 & 7 & $\begin{array}{l}\text { CC, EV, LIB, } \\
\text { TD, TMS }\end{array}$ & $\begin{array}{l}\text { This article presents a } \\
\text { thorough examination of } \\
\text { the current status of Li-ion } \\
\text { battery technology, } \\
\text { covering basics, } \\
\text { architectures, and overall } \\
\text { performance evaluation. }\end{array}$ & $\begin{array}{l}\text { - Short battery life and } \\
\text { limited load capacity. } \\
\text { High cost and } \\
\text { marginal safety. } \\
\text { - Small voltage and low } \\
\text { energy density. }\end{array}$ \\
\hline
\end{tabular}


Table 4. Cont.

\begin{tabular}{|c|c|c|c|c|c|c|}
\hline Rank & Ref. & $\mathrm{ACY}$ & $\begin{array}{c}\text { Citation Rank } \\
\text { Based on Table } 2\end{array}$ & $\begin{array}{l}\text { Abbreviated } \\
\text { Keywords }\end{array}$ & Contributions & $\begin{array}{c}\text { Research Gaps/Future } \\
\text { Directions }\end{array}$ \\
\hline 6 & {$[64]$} & 44.4 & 4 & $\begin{array}{l}\text { EL, } \\
\text { Forgetting factor, } \\
\text { Kullback-Leibler } \\
\text { divergence, } \\
\text { PM, RL }\end{array}$ & $\begin{array}{l}\text { A dynamic degradation } \\
\text { model for the } \mathrm{LiFePO}_{4} \\
\text { battery is developed to } \\
\text { quantitatively examine the } \\
\text { impact of different control } \\
\text { techniques in terms of } \\
\text { minimizing battery } \\
\text { deterioration. }\end{array}$ & $\begin{array}{l}\text { The calculation of the } \\
\text { number of battery cells } \\
\text { and SC modules in } \\
\text { obtaining accurate HESS } \\
\text { sizing is challenging. }\end{array}$ \\
\hline 7 & {$[66]$} & 34.8 & 6 & EV, EMS, HESS & $\begin{array}{l}\text { This research explores the } \\
\text { available literature on two } \\
\text { levels: the cell level and } \\
\text { the level of the } \\
\text { battery module. }\end{array}$ & $\begin{array}{l}\text { - Heat transfer } \\
\text { enhancement is not } \\
\text { always the greatest } \\
\text { option for dealing with } \\
\text { temperature } \\
\text { inconsistency. }\end{array}$ \\
\hline 8 & {$[68]$} & 30.4 & 8 & $\begin{array}{l}\text { EV, EM, ES, } \\
\text { Optimization, } \\
\text { Real-time }\end{array}$ & $\begin{array}{l}\text { The study discusses } \\
\text { real-time EMS for EVs } \\
\text { with HESS that includes a } \\
\text { battery and supercapacitor. }\end{array}$ & $\begin{array}{l}\lambda \text {-control is } \\
\text { better-suitable for } \\
\text { a high supercapacitor } \\
\text { voltage range. }\end{array}$ \\
\hline 9 & [74] & 26 & 15 & $\begin{array}{l}\text { BMS, ES, EV, } \\
\text { LIB, SOC }\end{array}$ & $\begin{array}{l}\text { The paper develops a } \\
\text { hardware prototype to } \\
\text { execute building energy } \\
\text { management and an } \\
\text { EV-charging } \\
\text { scheduling algorithm. }\end{array}$ & $\begin{array}{l}\text { The effects of PV output } \\
\text { and electricity } \\
\text { consumption forecast } \\
\text { errors along with the } \\
\text { vehicle-to-grid } \\
\text { performance should } \\
\text { be addressed in } \\
\text { future research. }\end{array}$ \\
\hline 10 & {$[70]$} & 21.6 & 10 & $\begin{array}{l}\text { Eco-driving, EV, } \\
\text { Optimal control }\end{array}$ & $\begin{array}{l}\text { This study investigates } \\
\text { EMS issues in EVs that } \\
\text { conform with online } \\
\text { standards for eco-driving. }\end{array}$ & $\begin{array}{l}\text { - In future research, this } \\
\text { method can be } \\
\text { implemented in real-time } \\
\text { EV applications to } \\
\text { provide online assistance } \\
\text { to the driver. }\end{array}$ \\
\hline
\end{tabular}

Table 5 demonstrates the categories of the manuscripts that were selected as the most relevant. The category of experimental works, development studies, and performance assessments has the most articles $(62.62 \%)$, followed by systematic and non-systematic reviews $(16.16 \%)$, and problem formulations and simulation analyses $(9 \%)$. There is a correlation between research categories, year ranges, and citation ranges. The largest number of publications (62) from the specified Scopus database of the most relevant articles, with a citation range (1-222), are focused on experimental work, development, and performance assessment-based analysis during the eight-year period up to 2021. From 2016 through 2021, the most common types of articles were technical overviews, observational articles, problem-formulation articles, and simulation analyses. 
Table 5. Types of the most relevant 100 manuscripts in Scopus database.

\begin{tabular}{cccc}
\hline Study Types & $\begin{array}{c}\text { Numer of } \\
\text { Manuscripts }\end{array}$ & Year Range & Citation Range \\
\hline $\begin{array}{c}\text { Experimental work, development, and } \\
\text { performance assessment }\end{array}$ & 62 & $2010-2021$ & $1-222$ \\
\hline Review (systematic/nonsystematic) & 16 & $2011-2021$ & $0-673$ \\
\hline $\begin{array}{c}\text { Problem formulation and } \\
\text { simulation analysis }\end{array}$ & 9 & $2016-2021$ & $0-187$ \\
\hline State of the art technical overview & 7 & $2014-2021$ & $0-384$ \\
\hline Case study, meta-analysis, and survey & 4 & $2016-2019$ & $7-163$ \\
\hline Technical and observational overview & 3 & $2012-2018$ & $11-212$ \\
\hline
\end{tabular}

The most common keywords from the selected 100 most relevant papers are listed as follows, along with most recent articles using these keywords: Energy Management Systems [79,163-165], Electric Vehicles [166,167], Secondary Batteries [168,169], Charging (batteries) [170], Energy Efficiency [171,172], Hybrid Energy Storage Systems [84,167,173], Plug-in Hybrid Vehicles [167], Fuzzy Logic [174], Model Predictive Control [175], Optimization [159,176], Controllers [61,109], DC-DC Converters [177], Stochastic Systems [178], Electric Power Transmission Networks [179,180], and State of Charge [181-183].

\subsection{Bibliometric Evaluation of Journals, Publishers, and Countries}

Figure 6 shows the proportion of papers published by thirteen individual publishers. Elsevier published the greatest proportion of articles among the selected papers (42\%). IEEE occupy the second position with $21 \%$, followed by Multidisciplinary Digital Publishing Institute with $17 \%$, the John Wiley \& Sons, Inc. with $7 \%$, and Inderscience Publishers with $2 \%$. The remaining papers were published by SAGE Publishing (1\%), Oxford University Press (1\%), ASME (1\%), Emerald Group (1\%), SAE International (1\%), IOS Press (1\%), Springer (1\%), and the Korean Institute of Power Electronics (1\%). Researchers are attempting to create new technologies and techniques that may be an alternative for the existing conventional fossil-based vehicle system because of their significant potential positive environmental impacts as well as the current research emphasis focusing mostly on EVs. Refs $[77,85,88,108,134,152,163]$ investigate models based on EMSs for EV applications.

The numbers of manuscripts published in the various journals and their impact factors (IF) are shown in Figure 7. All 100 of the most popular articles were published in 42 different publications. The top five journals with the largest number of publications published $46.46 \%$ of the 100 most relevant articles, with impact factors ranging from 0.975 to 14.982 . The "Energies" journal published the most papers (16), followed by "Applied Energy" with 10 articles; nevertheless, "Energy" and "International Journal of Energy Research" both published nine and six articles, respectively. The Renewable and Sustainable Energy Reviews journal contained five articles from the selected database. The journals "IEEE Access", "IEEE Transactions on Control Systems Technology", and "Journal of Power Sources" each contained four manuscripts. The impact factors of these journals ranged from 0.975 to 14.982 , according to the Journal Citations Report 2020. The journal "Renewable and Sustainable Energy Reviews" had the highest IF of 14.982. In comparison, with the same rate of publishing, the "IEEE Transactions on Vehicular Technology" journal had the lowest IF of 5.978 . 


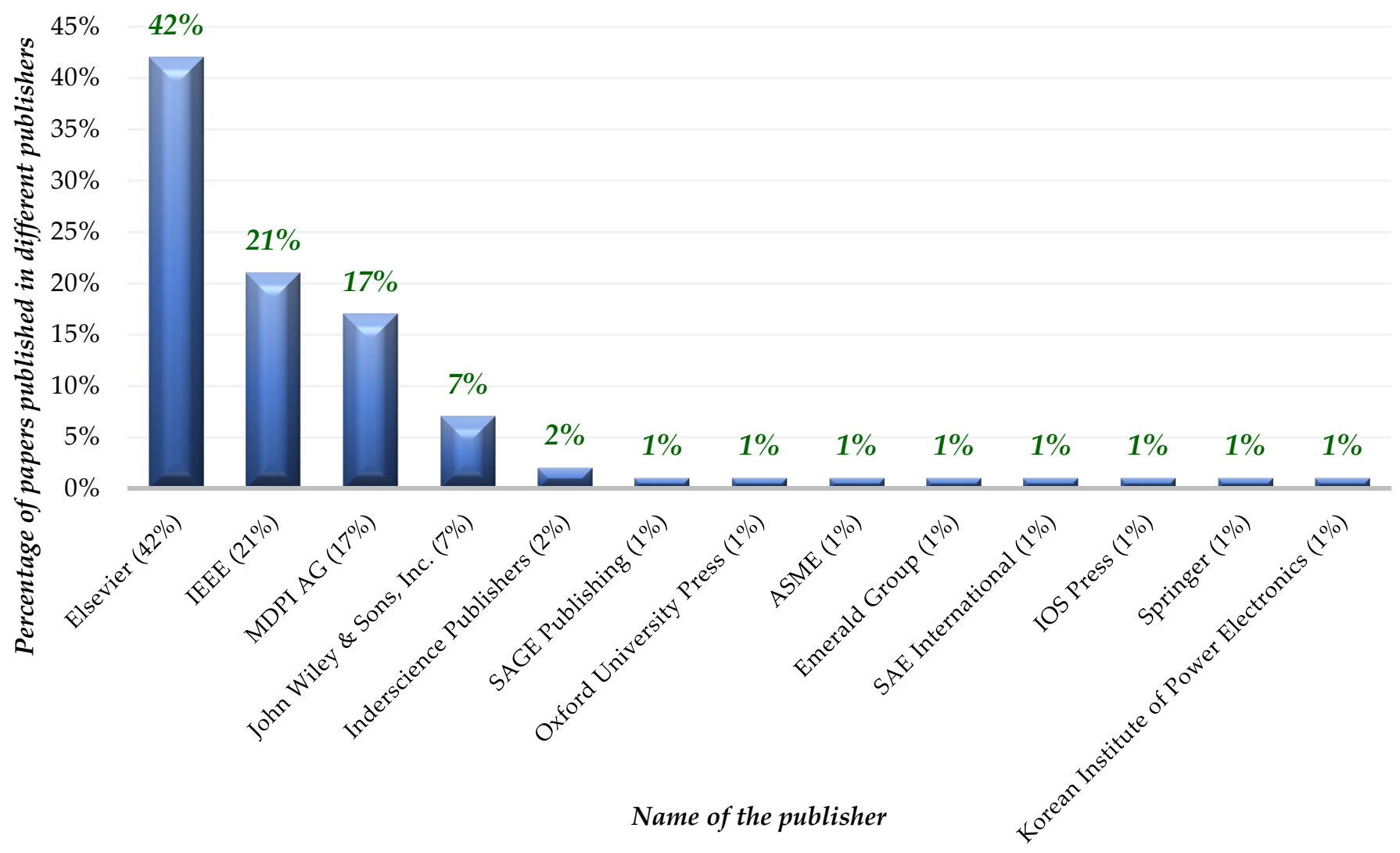

Figure 6. Distribution of 100 manuscripts across the different publishers.

\subsection{Document Authorship Analysis}

Table 6 represents the details of authors who published three or more papers. From the top 100 most relevant papers, 10 authors contributed more than three articles. With five manuscripts from the 100 most relevant manuscripts retrieved from the Scopus database, Zheng Chen of the Queen Mary University of London, United Kingdom, is the author with the most publications. Zheng Chen has an h-index of 25 with a total of 2687 citations. With four manuscripts, M.A. Hannan placed in the second position. His current affiliation is Universiti Tenaga Nasional, Malaysia, with an h-index of 40. With the same number of articles, Yonggang Liu, whose current affiliation is Chongqing University, China, gained the third position. The rest of the authors-Hongwen He, Md Murshadul Hoque, Guang Li, Jiangqiu Li, Azah Mohamed, Minggao Ouyang, and Yuanjian Zhang-produced three articles. China has the most authors $(n=5)$, whereas the United Kingdom and Malaysia have three and two, respectively. Minggao Ouyang of the Tsinghua University, China, has the most citations $(19,265)$ and the highest h-index $(68)$, followed by Jiangqiu Li and Azah Mohamed with 10,896 and 10,056 citations, respectively. They are from Tsinghua University, China, and Universiti Kebangsaan Malaysia, respectively. In contrast, the lowest citation (209) number was obtained by Yuanjian Zhang from Queen's University Belfast, United Kingdom, with an h-index of 9. Figure 8 represents the co-authorship analysis conducted using VOSviewer. The highest number of authors who participated in these selected manuscripts are from China, followed by the USA. 
(a)

(b)

\section{Name of journal}

IEEE Industrial Electronics Magazine

Energy for Sustainable Development

Electrical Engineering

Electric Power Systems Research

Control Engineering Practice

Computer Journal

Applied Thermal Engineering

Aircraft Engineering and Aerospace Technology

Journal of Energy Storage

Journal of Cleaner Production

Energy Conversion and Management

Journal of Power Sources

IEEE Transactions on Control Systems Technology

IEEE Access

Renewable and Sustainable Energy Reviews

IEEE Transactions on Vehicular Technology

International Journal of Energy Research

Energy

Applied Energy

Energies

\section{Publication frequency}

$\square 1$

1

1

1

1

1

$\square 1$

1

1

2
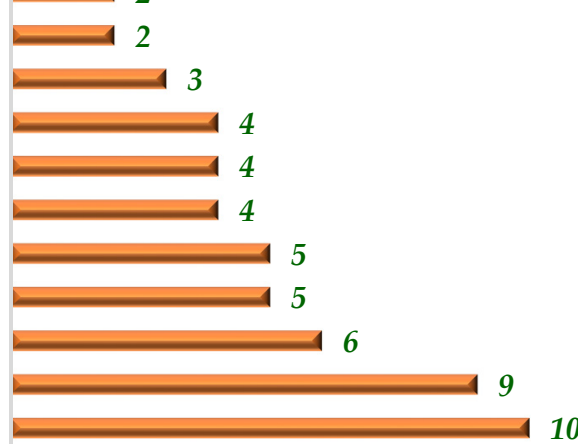

10

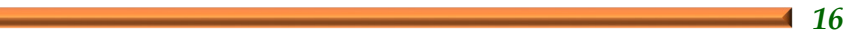

$\begin{array}{llllll}0 & 3 & 6 & 9 & 12 & 15\end{array}$

18

Impact Factor

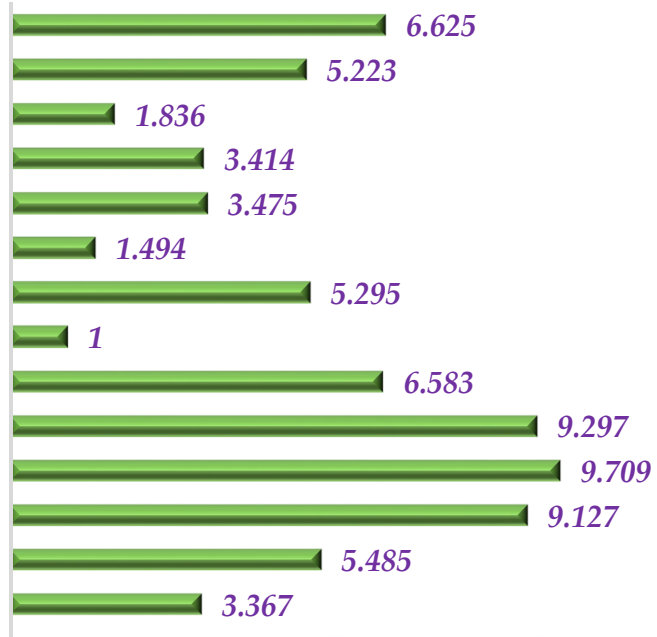

5.978

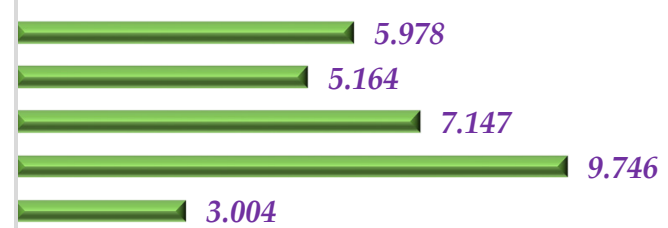

$\begin{array}{lllllllllllllllll}0 & 1 & 2 & 3 & 4 & 5 & 6 & 7 & 8 & 9 & 10 & 11 & 12 & 13 & 14 & 15 & 16\end{array}$

Figure 7. Distribution of manuscripts in terms of (a) journal evaluation and (b) journal impact factor. 
Table 6. Topmost 10 author profiles based on the number of manuscripts.

\begin{tabular}{|c|c|c|c|c|c|c|c|}
\hline Rank & Author Name & $\begin{array}{c}\text { Current } \\
\text { Affiliation }\end{array}$ & $\begin{array}{l}\text { Country } \\
\text { Name }\end{array}$ & $\begin{array}{l}\text { Number of } \\
\text { Manuscripts }\end{array}$ & $\begin{array}{l}\text { Total Number } \\
\text { of Citation }\end{array}$ & h-Index & $\begin{array}{l}\text { Authors } \\
\text { Position }\end{array}$ \\
\hline 1 & Chen, Zheng & $\begin{array}{c}\text { Queen Mary } \\
\text { University of } \\
\text { London }\end{array}$ & $\begin{array}{l}\text { United } \\
\text { Kingdom }\end{array}$ & 5 & 2687 & 25 & $\begin{array}{c}\text { First author }=2 \\
\text { Senior author }=1 \\
\text { Co-author }=2\end{array}$ \\
\hline 2 & Hannan, M. A. & $\begin{array}{l}\text { Universiti } \\
\text { Tenaga } \\
\text { Nasional }\end{array}$ & Malaysia & 4 & 7192 & 40 & $\begin{array}{c}\text { First author }=3 \\
\text { Co-author }=1\end{array}$ \\
\hline 3 & Liu, Yonggang & $\begin{array}{l}\text { Chongqing } \\
\text { University }\end{array}$ & China & 4 & 1061 & 18 & $\begin{array}{c}\text { First author }=2 \\
\text { Senior author }=2\end{array}$ \\
\hline 4 & He, Hongwen & $\begin{array}{l}\text { Beijing } \\
\text { Institute of } \\
\text { Technology }\end{array}$ & China & 3 & 8708 & 42 & Co-author $=3$ \\
\hline 5 & $\begin{array}{l}\text { Hoque, Md } \\
\text { Murshadul }\end{array}$ & $\begin{array}{l}\text { Monash } \\
\text { University }\end{array}$ & Australia & 3 & 1163 & 11 & $\begin{array}{c}\text { First author }=1 \\
\text { Co-author }=2\end{array}$ \\
\hline 6 & Li, Guang & $\begin{array}{l}\text { Queen Mary } \\
\text { University of } \\
\text { London }\end{array}$ & $\begin{array}{l}\text { United } \\
\text { Kingdom }\end{array}$ & 3 & 1219 & 21 & Co-author $=3$ \\
\hline 7 & Li, Jiangqiu & $\begin{array}{l}\text { Tsinghua } \\
\text { University }\end{array}$ & China & 3 & 10,896 & 53 & Co-author $=3$ \\
\hline 8 & $\begin{array}{l}\text { Mohamed, } \\
\text { Azah }\end{array}$ & $\begin{array}{l}\text { Universiti } \\
\text { Kebangsaan } \\
\text { Malaysia }\end{array}$ & Malaysia & 3 & 10,056 & 42 & $\begin{array}{c}\text { Senior author }=1 \\
\text { Co-author }=2\end{array}$ \\
\hline 9 & $\begin{array}{l}\text { Ouyang, } \\
\text { Minggao }\end{array}$ & $\begin{array}{l}\text { Tsinghua } \\
\text { University }\end{array}$ & China & 3 & 19,265 & 68 & $\begin{array}{c}\text { Senior author }=1 \\
\text { Co-author }=2\end{array}$ \\
\hline 10 & $\begin{array}{l}\text { Zhang, } \\
\text { Yuanjian }\end{array}$ & $\begin{array}{l}\text { Queen's } \\
\text { University } \\
\text { Belfast }\end{array}$ & $\begin{array}{l}\text { United } \\
\text { Kingdom }\end{array}$ & 3 & 209 & 9 & $\begin{array}{c}\text { First author }=1 \\
\text { Co-author }=2\end{array}$ \\
\hline
\end{tabular}

It was found that different authors have distinct fields of study. Zheng Chen of the Queen Mary University of London, United Kingdom, is primarily interested in Plug-in Hybrid Vehicles, Powertrains, and Energy Management [184-187]. He also wrote two manuscripts about EV applications, "Prediction of vehicle driving conditions with the incorporation of stochastic forecasting and machine learning and a case study in energy management of plug-in hybrid electric vehicles" [187] and "Stage of Charge Estimation of Lithium-Ion Battery Packs Based on Improved Cubature Kalman Filter with Long ShortTerm Memory Model" [186]. M.A. Hannan from Universiti Tenaga Nasional, Malaysia, developed the following recent manuscripts on EMSs for EV applications: "Techno-Economic Analysis and Environmental Impact of Electric Buses" [188], "Fuzzy Based ChargingDischarging Controller for Lithium-ion Battery" [189], and "Energy Storage Integrated Microgrid Performance Enhancement" [190]. Yonggang Liu from Chongqing University, China, has a primary research interest in Plug-in Hybrid Vehicles, Powertrains, and Energy Management [191-193]. "A survey on key techniques and development perspectives of equivalent consumption minimization strategy for hybrid electric vehicles" [193] and "Prediction of vehicle driving conditions with the incorporation of stochastic forecasting and machine learning and a case study in energy management of plug-in hybrid electric vehicles" [187] are the manuscripts recently produced by Yonggang Liu.

Figures 9 and 10 represent a graphical representation of the top ten countries that dominate the EMSs for the EV applications, and co-occurrence country analysis performed using VOSviewer, respectively. China is in the leading position based on the number of manuscripts in EMSs for EV applications, with a total of 44, followed by the USA, with 16 . With 12 manuscripts, France is in third place. Figure 10 also demonstrates that China has 
the highest number of collaborations with other countries. Regarding funding sponsors, the "National Natural Science Foundation of China" is in the first position with 22 sponsors, whereas the "National Key Research and Development Program of China" and the "China Scholarship Council" sponsored 8 and 7 manuscripts, respectively.

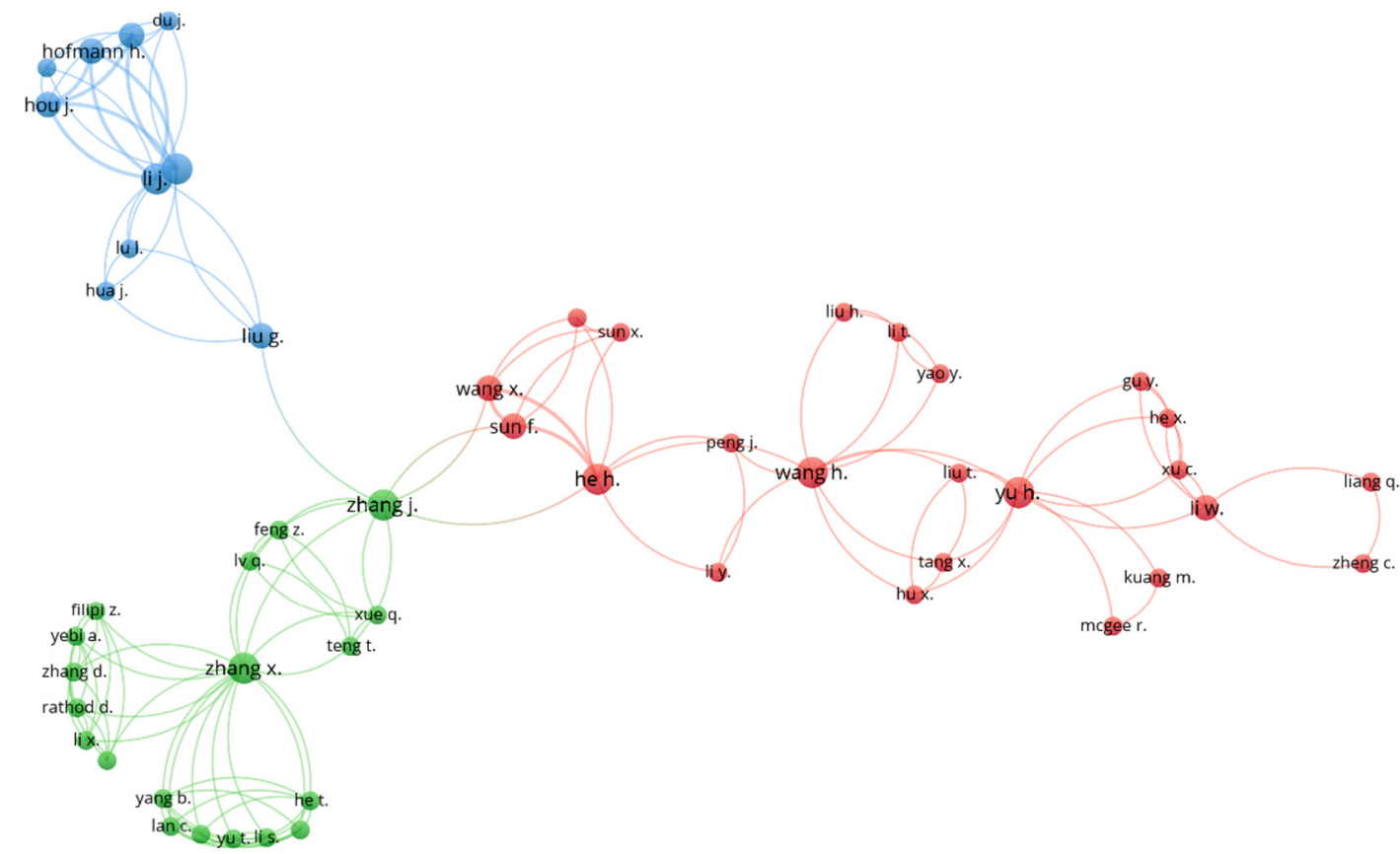

\& VoSviewer

Figure 8. Co-authorship analysis by VOSviewer from the Scopus database.

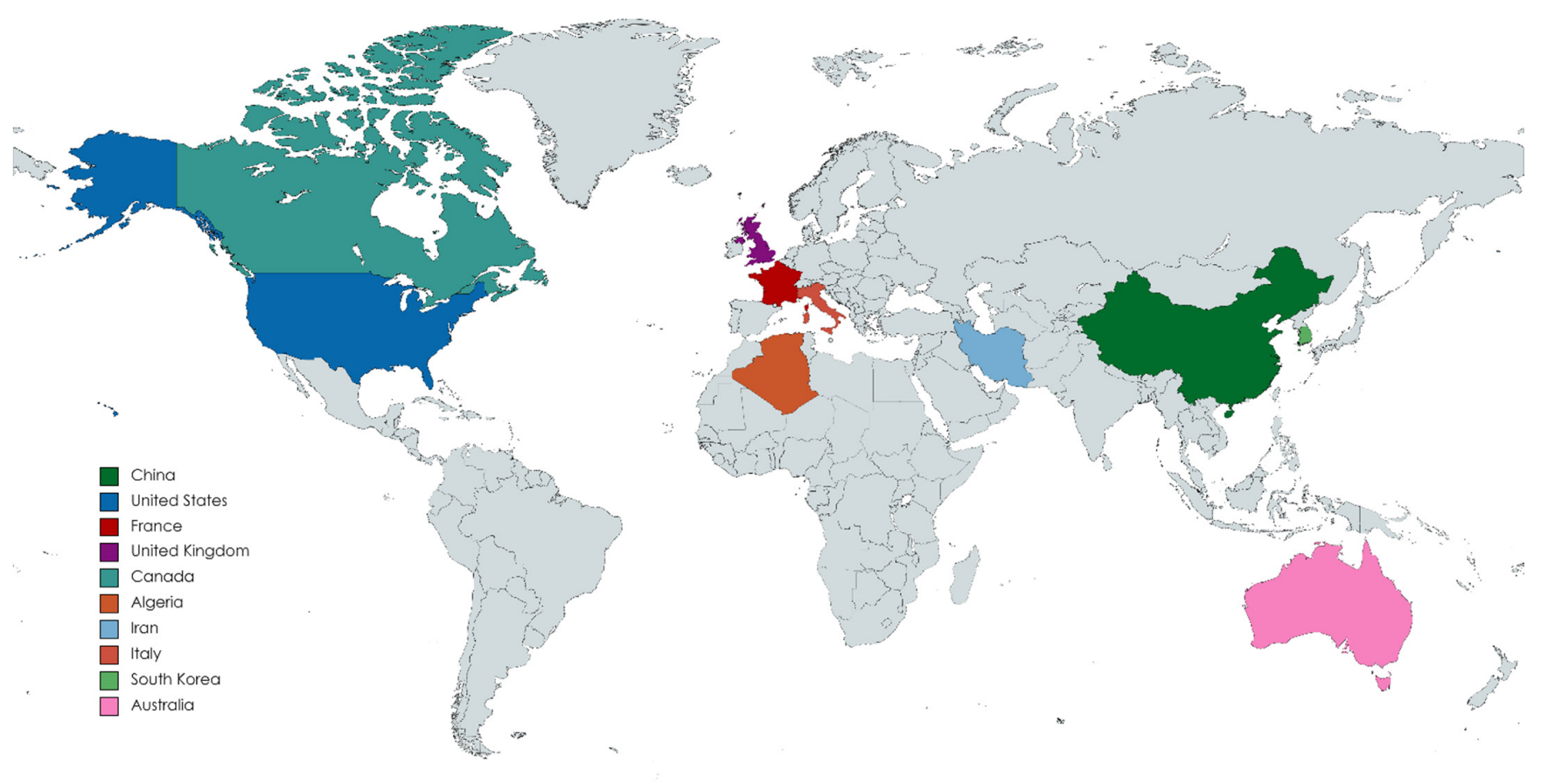

Figure 9. Graphical representation of top ten countries that dominate the EMSs for EV application. 


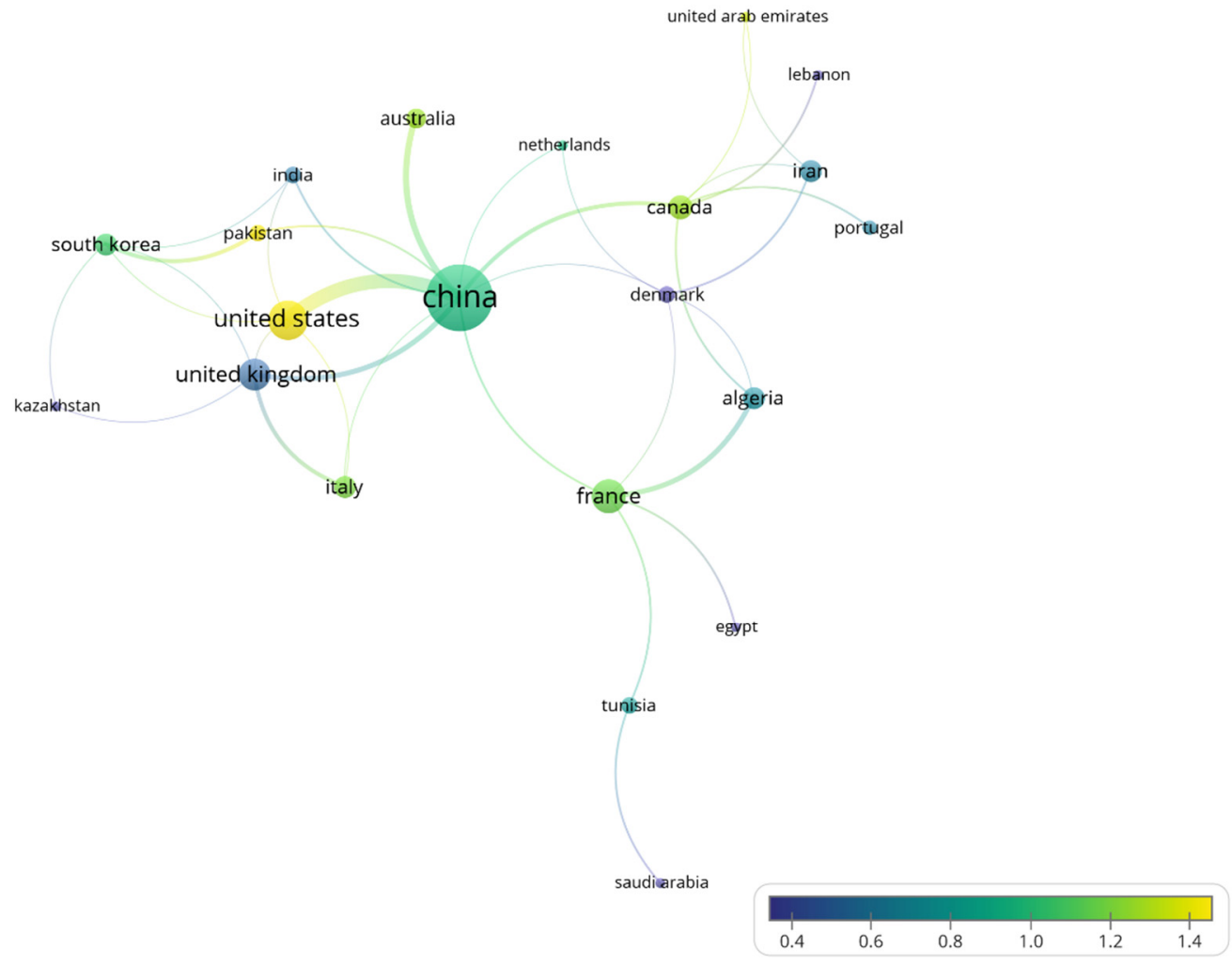

Figure 10. Co-occurrence country analysis of the Scopus database conducted using VOSviewer.

Following the discussion and analysis of the topmost 100 most relevant manuscripts from the chosen database, it was discovered that there had been a trend toward publishing both "review" and "technical" papers in recent years. "Energy Management Systems", "Electric Vehicles", "Secondary Batteries", "Energy Efficiency", "Hybrid Energy Storage Systems", and "Plug-in Hybrid Vehicles" are six areas of study that are gaining high interest. Different researchers have performed various types of literature evaluations and techniques in the fields of economic advantages of EV applications, EMSs, optimization and control for cost reduction, the flexibility of system operations, and the reducing of carbon emissions [77,85,88,108,134,141,144,152,163,194,195].

\section{Issues and Challenges of EMSs in EVs}

The EV applications have many key components that influence the EMS's dependability and efficiency. However, to overcome the problems of HEV EMS reliability and efficiency, several factors must be solved. The difficulties and challenges on EMSs for EV applications are split into the following categories and explored in the following sub-sections.

\subsection{Optimal EV Design and Power Distribution Challenges}

In ref $[142,149,152,153,155]$, the authors explored various issues related to optimal EV design and power distribution. However, the power flow and EV design need further improvement. Managing the power flow and satisfying the market's high expectations are critical in vehicle energy management systems that use a hybrid or a mix of several energy sources. The configuration and controller design are two major issues because of the complexity and difficulty of the integration needed among the other current systems in the vehicle [196]. When the EMSs are intended to reduce hydrogen consumption and enhance the fuel cell's life expectancy, which relates to design optimization, power distribution among various components becomes more difficult. Power splits have become a major worry due to frequent charging from the fuel cell to the battery and variations in fuel cell output power, both of which have an impact on the battery and fuel cell's performance 
and service life $[197,198]$. To ensure the dependability of EV applications, the EMSs must be further studied and improved in terms of hardware or real-time applications.

\subsection{Battery Thermal Management Issues}

In $[142,146,149,157,158]$, various authors discussed the issues concerning the thermal management of batteries. High temperature is generated because of chemical reactions, and this is a major issue that affects all batteries. Unusual temperatures harm the chemical characteristics of batteries and cause significant reductions in their efficiency. The temperature control system is also crucial for secondary batteries. In most cases, the battery must function at both low and high temperatures. Due to the lowered rate of chemical reactions and the transformation of active chemicals due to temperature, the charging and discharging currents and the battery's power handling capabilities are reduced by the impact of the low temperature [199]. On the contrary, the higher temperature in the battery creates certain challenging circumstances that induce aberrant chemical behavior and eventually lead to the battery exploding. Although some power may be saved by stimulating processes with the Arrhenius effect, a higher current creates a higher temperature, which leads to thermal runaway owing to positive temperature feedback. To avoid the battery's thermal runaway, specific measures must be implemented. Compared to other popular batteries, the capacity of the Li-ion battery increases as the temperature rises at the expense of the battery's life. Therefore, further attention is needed to address the battery's thermal issues to achieve better efficiency.

\subsection{Battery Storage Life Cycle and Aging Issues}

In $[142,145,148,149,151,154-156]$, numerous authors investigated the battery storage life cycle and aging issues. The loss of battery cell function due to voltage and heat impacts has been studied; nevertheless, this loss reduces the battery's longevity. It's worth noting that running a battery cell outside its normal working range results in irreversible capacity loss. This anomaly has a cumulative impact, reducing battery life and possibly causing total and irreversible loss of usage [200]. Owing to anode plating, the lifespan reduces slowly at low temperatures (below $10^{\circ} \mathrm{C}$ ), but it drops dramatically at high temperatures (over $60^{\circ} \mathrm{C}$ ) due to chemical breakdown. As a result, the optimal operating temperature range should neither be too large nor too narrow. Hence, further investigation is suggested, particularly the conducting of feasibility studies to reduce the impact of cell function on battery life.

\subsection{Power Electronic Controller and Converter Issues}

In $[90,102,123,139]$, different authors examined power electronics controller and converter issues including switching loss, high ripple current, voltage stress, high voltage gain, high impedance, optimization integration, and complex control techniques. The fuel cell generates low DC voltage by regulating the fuel cell to a level with the DC bus voltage through an appropriate DC-DC converter [201] with three distinct energy sources. As a result, in an EV, a power electronic interface is required. Open circuit failures pull increments of fuel cell stack ripple currents and add additional stress to inductors; thus, precautions must be taken before its growth. Driver failure, improper gate voltage, device damage, and excessive voltage, current, and transients are possible problems [202]. The DC-DC converter in the fuel cell system must be boosted, and the storage device requires a bidirectional DC-DC converter [203]. As a result, for the battery and SC, buck-boost converters are commonly employed [204]. Therefore, further studies are required to develop an efficient controller and converter for EV applications.

\subsection{Environmental and Decarbonization Issues}

In [145], Torabi et al. focused on the environmental and decarbonization issues for EVs. Further research should be conducted on the impacts of EVs in terms of reducing carbon emissions. Automobile electrification, such as EVs, HEVs, and PHEVs, is becoming 
more popular as oil costs rise and the demand for large amounts of energy for sustainable transportation grows. Toyota estimated that by 2020, EVs would account for more than $7 \%$ of global transportation [205]. Li-ion batteries generate $\mathrm{CO}_{2}$ and GHGs during their manufacturing and disposal, despite their positive influence on the environment by lowering the number of oil-based cars [206]. In a previous study, the US EPA examined Li-ion batteries for their use of nickel- and cobalt-based cathodes, as well as solvent-based electrode processing, and found significant environmental impacts, such as resource depletion, global warming, ecological toxicity, and human health effects, among other things [207]. According to this study, people involved in the manufacturing, processing, and use of cobalt and nickel metal compounds may be at risk for respiratory, pulmonary, and neurological disorders [207]. This danger can be mitigated by using a Li-ion battery recycling method to save natural resources and minimize the usage of nickel and cobalt $[207,208]$. Thus, the impact of EVs on the environment and towards achieving sustainable development goals (SDG) needs to be further enhanced.

\subsection{Standard Regulation and Policy Issues}

Policy and regulation are important issues in EV industries. In [82], Liu et al. discussed various regulations and policy-related issues. Clean development mechanisms (CDM), carbon trading (CT), and joint implementation (JT) are the three main elements within the Kyoto Protocol that the UN has defined to cost-effectively meet their emission reduction objectives [209]. Many measures have been implemented to decarbonize Europe's electricity industry. By utilizing a linear dynamic optimization model, the economic implications of the alternative energy strategy for Europe's power sector to cut greenhouse gas emissions by 80 to $95 \%$ by 2050 as compared to 1990 were calculated [210]. By 2050, Europe will convert to a $100 \%$ renewable energy system using the energy system transition model developed by Lappeenranta University of Technology [211]. Although a few effective initiatives have been undertaken to promote EVs, the long-term planning of EV use, including standardization, laws, enforcement, regulation, financial incentives, and policies, needs further attention.

\section{Future Trends of EMSs in EV Applications}

Based on a rigorous review of the existing notable articles, this bibliometric study delivered several major and selective proposals for future research towards the advancement of EMSs in EV applications.

- The global acceptance of EMSs in EV applications was discussed in terms of achieving SDG in the transportation sector. Nonetheless, various issues related to EMSs in EV applications, such as short driving ranges, battery lifetimes, long charging times, high initial costs, poor vehicles, and ineffective EV-based policies, need to be carefully examined. Further research is recommended to develop an efficient EMSs design with better operational mechanisms, encouraging market regulations and global collaborations for efficient EV operations.

- The existing converter designs implemented in EMSs suffer from various issues such as current stress, low impedance, high ripple current, and sensitive duty cycles. In this regard, further investigations are needed to optimize the converter design to achieve high frequency and low losses. Additionally, optimization based on mechanical design is suggested to obtain high robustness, mechanical strength, and power density.

- The application of enhanced control techniques towards achieving various benefits such as bidirectional power management, fast-tracking, and high efficiency can be observed in EMSs. Nevertheless, the implemented control technique suffers from various disadvantages such as lengthy training durations, computational complexity, and suitable hyperparameter adjustment. Therefore, further exploration is required to address control technique issues.

- Due to the implementation of EMSs in EV application for controlling battery heating and cooling, the reliability and stability of battery operation are improved significantly. 
However, the efficiency of EVs is reduced due to the existence of thermal issues and deep-diving range loss. Additionally, the occurrence of thermal effects due to the electrochemical process results in poor EMSs accuracy and stability. To minimize the dynamic instability issues, the utilization of super capacitors in the battery storage system can be observed. Additionally, the optimization scheme in EMSs technology could effectively reduce battery aging and power curtailment issues.

- The performance of EMSs in EV applications can be improved by accurately estimating various states of batteries, such as SOC, SOH, and RUL, respectively. The inaccurate measurement of battery SOC would result in charging issues. Further, the inappropriate measurement of SOH and RUL would lead to early replacement of batteries, delays in battery replacement, explicit failure events, and further increases in cost. Therefore, further investigation should be conducted regarding the application of deep learning techniques for better estimation accuracy. Additionally, the application of multi-scale and co-estimation techniques in BMS technology would increase efficiency and minimize computational cost.

- The implementation of algorithm hybridization schemes was shown to be beneficial, with better accuracy and effectiveness than non-hybridized techniques. The development of the hybridization technique takes place by performing the integration of two or more intelligent techniques. The hybridized intelligent techniques may comprise an integrated intelligent algorithm with an optimization model or a combination of two intelligent models. However, hybridization schemes suffer from operational complexity and long training times, and they require human expertise and high computational processors to conduct the desired operations. Hence, further explorations, which aim to develop an efficient hybrid model while considering practicability issues, are needed.

- Even though substantial progress towards SDG and decarbonization has been accomplished with EMS-based EV applications, environmental issues such as soil and groundwater contamination need to be considered. Inaccurate battery disposal would result in health hazards from water as well as air. To prevent inappropriate disposal of batteries in the environment, adequate measures in terms of recycling and reusability should be carried out.

- To improve the performance capability and robustness of EMSs in EV applications, the implementation of real-time monitoring consists of sensors, data processors, and cloudbased technology. The performance of EMSs in EVs can be observed by acquiring realtime data in the form of voltage, current, impedance, temperature, etc. Additionally, the state estimation of the battery can be performed and stored in the cloud database. The effectiveness of the EMSs can be improved with suitable data extraction, data processing, and prediction in real-time applications.

\section{Conclusions}

The use of advanced EMSs in EVs is essential to achieve optimal power distribution and improve the cost, efficiency, lifespan, and effectiveness of EV batteries with regard to battery monitoring and management systems, charge and discharge controls, state estimation, energy storage safety, and protection. Thus, this study identified the 100 most relevant publications on EMSs for EV applications from the Scopus' database to assess the recent trends, performance, applications, issues, and problems. Many studies were also presented, including the distribution of 15 popular keywords-in terms of the most cited articles-by year, nation, publisher, and journal, and the grouping of studies by research field and study type. The primary goal of this article was to provide an overview of academic research trends and recognize the features and progress of EMSs in EV applications identified in high-impact research publications. Many challenges and possible solutions were also discussed for the EMSs for the EV applications related to this article in terms of the achievement of system flexibility and system cost reduction for EV applications. 
The most relevant 100 articles were included in this bibliometric analysis to provide insights into the history, current approach of researchers in scientific investigation, and challenges related to EMSs for EV applications. There are several advantages to determining the characteristics of the most relevant publications, including:

- The systematic/non-systematic study and investigation of the most referenced manuscripts provide insights into the history and evolution that has shaped contemporary knowledge and practice.

- The characteristics of the most relevant articles in EMSs for EV applications can provide future researchers with a clear picture.

- The bibliographical analysis may give researchers an excellent perspective on a dynamic and expanding study area, inspiring various dedicated researchers to employ contemporary and new technologies to enhance a specific research field.

- $\quad$ Researchers and journal editors may use the most relevant article analysis to assist them in evaluating submitted manuscripts.

In conclusion, it is expected that valuable information, discussions, knowledge, and analysis on prominent EMS papers on EV applications between 2010 and 2021 would not only assist in enhancing EV operations but would also provide valuable guidelines and suggestions for automobile engineers and researchers towards achieving decarbonization targets and SDGs.

Author Contributions: Conceptualization, M.S.M. and M.S.H.L.; methodology, M.S.M.; formal analysis, M.S.M. and M.S.H.L.; investigation, M.S.M. and M.S.H.L.; resources, M.S.M. and M.S.H.L.; data curation, M.S.M.; writing—original draft preparation, M.S.M. and S.A.; writing—review and editing, M.S.H.L., K.H., T.J., H.M., R.M.E. and A.H.; visualization, M.S.M. and S.T.M.; supervision, M.S.H.L.; project administration, M.S.H.L.; funding acquisition, M.S.H.L. All authors have read and agreed to the published version of the manuscript.

Funding: This research was funded by Universiti Kebangsaan Malaysia under Grant Code GGPM-2020-006.

Institutional Review Board Statement: Not applicable.

Informed Consent Statement: Not applicable.

Data Availability Statement: Not applicable.

Conflicts of Interest: The authors declare no conflict of interest.

\section{References}

1. Thombre, A.; Agarwal, A. A Paradigm Shift in Urban Mobility: Policy Insights from Travel before and after COVID-19 to Seize the Opportunity. Transp. Policy 2021, 110, 335-353. [CrossRef]

2. Sumabat, A.K.; Lopez, N.S.; Yu, K.D.; Hao, H.; Li, R.; Geng, Y.; Chiu, A.S.F. Decomposition Analysis of Philippine CO2 Emissions from Fuel Combustion and Electricity Generation. Appl. Energy 2016, 164, 795-804. [CrossRef]

3. Santos, G. Road Transport and CO2 Emissions: What Are the Challenges? Transp. Policy 2017, 59, 71-74. [CrossRef]

4. Almeida, A.; Sousa, N.; Coutinho-Rodrigues, J. Quest for Sustainability: Life-Cycle Emissions Assessment of Electric Vehicles Considering Newer Li-Ion Batteries. Sustainability 2019, 11, 2366. [CrossRef]

5. Teoh, T.; Kunze, O.; Teo, C.C.; Wong, Y.D. Decarbonisation of Urban Freight Transport Using Electric Vehicles and Opportunity Charging. Sustainability 2018, 10, 3258. [CrossRef]

6. Hannan, M.A.; Hoque, M.M.; Mohamed, A.; Ayob, A. Review of Energy Storage Systems for Electric Vehicle Applications: Issues and Challenges. Renew. Sustain. Energy Rev. 2017, 69, 771-789. [CrossRef]

7. Hossain Lipu, M.S.; Hannan, M.A.; Karim, T.F.; Hussain, A.; Saad, M.H.M.; Ayob, A.; Miah, M.S.; Indra Mahlia, T.M. Intelligent Algorithms and Control Strategies for Battery Management System in Electric Vehicles: Progress, Challenges and Future Outlook. J. Clean. Prod. 2021, 292, 126044. [CrossRef]

8. Ghosh, A. Possibilities and Challenges for the Inclusion of the Electric Vehicle (EV) to Reduce the Carbon Footprint in the Transport Sector: A Review. Energies 2020, 13, 2602. [CrossRef]

9. Yaacob, N.F.F.; Yazid, M.R.M.; Maulud, K.N.A.; Basri, N.E.A. A Review of the Measurement Method, Analysis and Implementation Policy of Carbon Dioxide Emission from Transportation. Sustainability 2020, 12, 5873. [CrossRef]

10. Aki, H.; Wakui, T.; Yokoyama, R. Development of an Energy Management System for Optimal Operation of Fuel Cell Based Residential Energy Systems. Int. J. Hydrogen Energy 2016, 41, 20314-20325. [CrossRef] 
11. Tahri, A.; El Fadil, H.; Belhaj, F.Z.; Gaouzi, K.; Rachid, A.; Giri, F.; Chaoui, F.Z. Management of Fuel Cell Power and Supercapacitor State-of-Charge for Electric Vehicles. Electr. Power Syst. Res. 2018, 160, 89-98. [CrossRef]

12. Wu, W.; Partridge, J.S.; Bucknall, R.W.G. Simulation of a Stabilised Control Strategy for PEM Fuel Cell and Supercapacitor Hybrid Propulsion System for a City Bus. Int. J. Hydrogen Energy 2018, 43, 19763-19777. [CrossRef]

13. Wu, W.; Partridge, J.S.; Bucknall, R.W.G. Stabilised Control Strategy for PEM Fuel Cell and Supercapacitor Propulsion System for a City Bus. Int. J. Hydrogen Energy 2018, 43, 12302-12313. [CrossRef]

14. Payman, A.; Pierfederici, S.; Meibody-Tabar, F. Energy Control of Supercapacitor/Fuel Cell Hybrid Power Source. Energy Convers. Manag. 2008, 49, 1637-1644. [CrossRef]

15. Li, H.; Ravey, A.; N’Diaye, A.; Djerdir, A. A Novel Equivalent Consumption Minimization Strategy for Hybrid Electric Vehicle Powered by Fuel Cell, Battery and Supercapacitor. J. Power Sources 2018, 395, 262-270. [CrossRef]

16. Zhang, X.; Liu, L.; Dai, Y. Fuzzy State Machine Energy Management Strategy for Hybrid Electric UAVs with PV/Fuel Cell/Battery Power System. Int. J. Aerosp. Eng. 2018, 2018. [CrossRef]

17. Hames, Y.; Kaya, K.; Baltacioglu, E.; Turksoy, A. Analysis of the Control Strategies for Fuel Saving in the Hydrogen Fuel Cell Vehicles. Int. J. Hydrogen Energy 2018, 43, 10810-10821. [CrossRef]

18. Chettibi, N.; Mellit, A.; Sulligoi, G.; Massi Pavan, A. Adaptive Neural Network-Based Control of a Hybrid AC/DC Microgrid. IEEE Trans. Smart Grid 2018, 9, 1667-1679. [CrossRef]

19. Patel, R.; Deb, D. Parametrized Control-Oriented Mathematical Model and Adaptive Backstepping Control of a Single Chamber Single Population Microbial Fuel Cell. J. Power Sources 2018, 396, 599-605. [CrossRef]

20. Sankar, K.; Jana, A.K. Nonlinear Multivariable Sliding Mode Control of a Reversible PEM Fuel Cell Integrated System. Energy Convers. Manag. 2018, 171, 541-565. [CrossRef]

21. Ray, P.K.; Singh, V.P.; Mohanty, S.R.; Kishor, N.; Sen, S. Frequency Control Based on Ho Controller for Small Hybrid Power System. In Proceedings of the 2011 5th International Power Engineering and Optimization Conference, PEOCO 2011-Program and Abstracts, Shah Alam, Malaysia, 6-7 June 2011; pp. 227-232. [CrossRef]

22. Yang, F.; Sheng, B.; Fu, Y. Energy Management for Fuel Cell-Supercapacitor Hybrid System Using Passivity-Based Controller with Multi-Equilibrium States. In Proceedings of the IECON 2015-41st Annual Conference of the IEEE Industrial Electronics Society, Yokohama, Japan, 9-12 November 2015; pp. 511-516. [CrossRef]

23. Thounthong, P.; Piegari, L.; Pierfederici, S.; Davat, B. Nonlinear Intelligent DC Grid Stabilization for Fuel Cell Vehicle Applications with a Supercapacitor Storage Device. Int. J. Electr. Power Energy Syst. 2015, 64, 723-733. [CrossRef]

24. Garcia, P.; Fernandez, L.M.; Garcia, C.A.; Jurado, F. Energy Management System of Fuel-Cell-Battery Hybrid Tramway. IEEE Trans. Ind. Electron. 2010, 57, 4013-4023. [CrossRef]

25. MacKie, D.M.; Jahnke, J.P.; Benyamin, M.S.; Sumner, J.J. Simple, Fast, and Accurate Methodology for Quantitative Analysis Using Fourier Transform Infrared Spectroscopy, with Bio-Hybrid Fuel Cell Examples. MethodsX 2016, 3, 128-138. [CrossRef]

26. Pahon, E.; Yousfi Steiner, N.; Jemei, S.; Hissel, D.; Péra, M.C.; Wang, K.; Moçoteguy, P. Solid Oxide Fuel Cell Fault Diagnosis and Ageing Estimation Based on Wavelet Transform Approach. Int. J. Hydrogen Energy 2016, 41, 13678-13687. [CrossRef]

27. Thounthong, P.; Raël, S.; Davat, B. Energy Management of Fuel Cell/Battery/Supercapacitor Hybrid Power Source for Vehicle Applications. J. Power Sources 2009, 193, 376-385. [CrossRef]

28. Abdelshafy, A.M.; Hassan, H.; Jurasz, J. Optimal Design of a Grid-Connected Desalination Plant Powered by Renewable Energy Resources Using a Hybrid PSO-GWO Approach. Energy Convers. Manag. 2018, 173, 331-347. [CrossRef]

29. Suresh, R.; Sankaran, G.; Joopudi, S.; Choudhury, S.R.; Narasimhan, S.; Rengaswamy, R. Optimal Power Distribution Control for a Network of Fuel Cell Stacks. J. Process Control 2019, 74, 88-98. [CrossRef]

30. Ou, K.; Yuan, W.W.; Choi, M.; Yang, S.; Jung, S.; Kim, Y.B. Optimized Power Management Based on Adaptive-PMP Algorithm for a Stationary PEM Fuel Cell/Battery Hybrid System. Int. J. Hydrogen Energy 2018, 43, 15433-15444. [CrossRef]

31. Feroldi, D.; Carignano, M. Sizing for Fuel Cell/Supercapacitor Hybrid Vehicles Based on Stochastic Driving Cycles. Appl. Energy 2016, 183, 645-658. [CrossRef]

32. Li, T.; Liu, H.; Ding, D. Predictive Energy Management of Fuel Cell Supercapacitor Hybrid Construction Equipment. Energy 2018, 149, 718-729. [CrossRef]

33. Bortoluzzi, M.; Correia de Souza, C.; Furlan, M. Bibliometric Analysis of Renewable Energy Types Using Key Performance Indicators and Multicriteria Decision Models. Renew. Sustain. Energy Rev. 2021, 143, 110958. [CrossRef]

34. Choi, W.; Kim, J.; Lee, S.E.; Park, E. Smart Home and Internet of Things: A Bibliometric Study. J. Clean. Prod. 2021, $301,126908$. [CrossRef]

35. Tseng, M.-L.; Chang, C.-H.; Lin, C.-W.R.; Wu, K.-J.; Chen, Q.; Xia, L.; Xue, B. Future Trends and Guidance for the Triple Bottom Line and Sustainability: A Data Driven Bibliometric Analysis. Environ. Sci. Pollut. Res. 2020, 27, 33543-33567. [CrossRef]

36. Ismail, S.A.; Ang, W.L.; Mohammad, A.W. Electro-Fenton Technology for Wastewater Treatment: A Bibliometric Analysis of Current Research Trends, Future Perspectives and Energy Consumption Analysis. J. Water Process Eng. 2021, $40,101952$. [CrossRef]

37. Gingras, Y. Bibliometrics and Research Evaluation: Uses and Abuses; The MIT Press: Cambridge, MA, USA, 2016 ; pp. 1-115.

38. Andrés, A. Measuring Academic Research: How to Undertake a Bibliometric Study; Chandos Publishing: Oxford, UK, 2019; pp. 1-165.

39. Walsh, C.; Lydon, S.; Byrne, D.; Madden, C.; Fox, S.; O'Connor, P. The 100 Most Cited Articles on Healthcare Simulation: A Bibliometric Review. Simul. Healthc. 2018, 13, 211-220. [CrossRef] 
40. Ruttenstock, E.; Friedmacher, F.; Höllwarth, M.E.; Coran, A.G.; Puri, P. The 100 Most-Cited Articles in Pediatric Surgery International. Pediatr. Surg. Int. 2012, 28, 563-570. [CrossRef]

41. Baker, N.C.; Ekins, S.; Williams, A.J.; Tropsha, A. A Bibliometric Review of Drug Repurposing. Drug Discov. Today 2018, 23, 661-672. [CrossRef]

42. Yeo, W.; Kim, S.; Park, H.; Kang, J. A Bibliometric Method for Measuring the Degree of Technological Innovation. Technol. Forecast. Soc. Chang. 2015, 95, 152-162. [CrossRef]

43. Vogel, R.; Güttel, W.H. The Dynamic Capability View in Strategic Management: A Bibliometric Review. Int. J. Manag. Rev. 2013, 15, 426-446. [CrossRef]

44. Meerow, S.; Newell, J.P. Resilience and Complexity: A Bibliometric Review and Prospects for Industrial Ecology. J. Ind. Ecol. 2015, 19, 236-251. [CrossRef]

45. Belter, C.W.; Seidel, D.J. A Bibliometric Analysis of Climate Engineering Research. Wiley Interdiscip. Rev. Clim. Chang. 2013, 4, 417-427. [CrossRef]

46. Shukla, A.K.; Janmaijaya, M.; Abraham, A.; Muhuri, P.K. Engineering Applications of Artificial Intelligence: A Bibliometric Analysis of 30 Years (1988-2018). Eng. Appl. Artif. Intell. 2019, 85, 517-532. [CrossRef]

47. Cancino, C.; Merigó, J.M.; Coronado, F.; Dessouky, Y.; Dessouky, M. Forty Years of Computers \& Industrial Engineering: A Bibliometric Analysis. Comput. Ind. Eng. 2017, 113, 614-629. [CrossRef]

48. Ibrahim, A.-B.M.A.; Julius, R.; Choudhury, P.K. Progress in Quantum Electronics Research: A Bibliometric Analysis. J. Electromagn. Waves Appl. 2020, 35, 549-565. [CrossRef]

49. Wang, Q.; Yang, Z.; Yang, Y.; Long, C.; Li, H. A Bibliometric Analysis of Research on the Risk of Engineering Nanomaterials during 1999-2012. Sci. Total Environ. 2014, 473-474, 483-489. [CrossRef] [PubMed]

50. de Freitas, F.G.; de Souza, J.T. Ten Years of Search Based Software Engineering: A Bibliometric Analysis. Lecture Notes in Computer Science (including subseries Lecture Notes in Artificial Intelligence and Lecture Notes in Bioinformatics). In Proceedings of the 7th International Workshop on Programming Multi-Agent Systems, Budapest, Hungary, 10-15 May 2009; pp. 5-6. [CrossRef]

51. Ayodele, B.V.; Mustapa, S.I. Life Cycle Cost Assessment of Electric Vehicles: A Review and Bibliometric Analysis. Sustainability 2020, 12, 2387. [CrossRef]

52. Ramirez Barreto, D.A.; Ochoa Guillermo, E.V.; Peña Rodriguez, A.; Cardenas Escorcia, Y.d.C. Bibliometric Analysis of Nearly a Decade of Research in Electric Vehicles: A Dynamic Approach. ARPN J. Eng. Appl. Sci. 2018, 13, 1-7.

53. Zhao, X.; Wang, S.; Wang, X. Characteristics and Trends of Research on New Energy Vehicle Reliability Based on Theweb of Science. Sustainability 2018, 10, 3560. [CrossRef]

54. Wang, S.; Yu, J. A Bibliometric Research on Next-Generation Vehicles Using CiteSpace. Recycling 2021, 6, 14. [CrossRef]

55. Arriola, E.R.; Ubando, A.T.; Chen, W.-H. A Bibliometric Review on the Application of Fuzzy Optimization to Sustainable Energy Technologies. Int. J. Energy Res. 2020, 1-12. [CrossRef]

56. Tian, X.; Geng, Y.; Zhong, S.; Wilson, J.; Gao, C.; Chen, W.; Yu, Z.; Hao, H. A Bibliometric Analysis on Trends and Characters of Carbon Emissions from Transport Sector. Transp. Res. Part D Transp. Environ. 2018, 59, 1-10. [CrossRef]

57. Gandia, R.M.; Antonialli, F.; Cavazza, B.H.; Neto, A.M.; de Lima, D.A.; Sugano, J.Y.; Nicolai, I.; Zambalde, A.L. Autonomous Vehicles: Scientometric and Bibliometric Review. Transp. Rev. 2018, 39, 9-28. [CrossRef]

58. Zhang, L.; Liu, Y.; Pang, B. China's Development on New Energy Vehicle Battery Industry: Based on Market and Bibliometrics. IOP Conf. Ser. Earth Environ. Sci. 2020, 581, 012003. [CrossRef]

59. Cabeza, L.F.; Chàfer, M.; Mata, É. Comparative Analysis of Web of Science and Scopus on the Energy Efficiency and Climate Impact of Buildings. Energies 2020, 13, 409. [CrossRef]

60. Borri, E.; Tafone, A.; Zsembinszki, G.; Comodi, G.; Romagnoli, A.; Cabeza, L.F. Recent Trends on Liquid Air Energy Storage: A Bibliometric Analysis. Appl. Sci. 2020, 10, 2773. [CrossRef]

61. Hoque, M.M.; Hannan, M.A.; Mohamed, A.; Ayob, A. Battery Charge Equalization Controller in Electric Vehicle Applications: A Review. Renew. Sustain. Energy Rev. 2017, 75, 1363-1385. [CrossRef]

62. Hannan, M.A.; Lipu, M.S.H.; Hussain, A.; Mohamed, A. A Review of Lithium-Ion Battery State of Charge Estimation and Management System in Electric Vehicle Applications: Challenges and Recommendations. Renew. Sustain. Energy Rev. 2017, 78, 834-854. [CrossRef]

63. Rahimi-Eichi, H.; Ojha, U.; Baronti, F.; Chow, M.Y. Battery Management System: An Overview of Its Application in the Smart Grid and Electric Vehicles. IEEE Ind. Electron. Mag. 2013, 7, 4-16. [CrossRef]

64. Xiong, R.; Cao, J.; Yu, Q. Reinforcement Learning-Based Real-Time Power Management for Hybrid Energy Storage System in the Plug-in Hybrid Electric Vehicle. Appl. Energy 2018, 211, 538-548. [CrossRef]

65. Hannan, M.A.; Hoque, M.M.; Hussain, A.; Yusof, Y.; Ker, P.J. State-of-the-Art and Energy Management System of Lithium-Ion Batteries in Electric Vehicle Applications: Issues and Recommendations. IEEE Access 2018, 6, 19362-19378. [CrossRef]

66. Song, Z.; Hofmann, H.; Li, J.; Hou, J.; Han, X.; Ouyang, M. Energy Management Strategies Comparison for Electric Vehicles with Hybrid Energy Storage System. Appl. Energy 2014, 134, 321-331. [CrossRef]

67. Xia, G.; Cao, L.; Bi, G. A Review on Battery Thermal Management in Electric Vehicle Application. J. Power Sources 2017, 367, 90-105. [CrossRef] 
68. Castaings, A.; Lhomme, W.; Trigui, R.; Bouscayrol, A. Comparison of Energy Management Strategies of a Battery/Supercapacitors System for Electric Vehicle under Real-Time Constraints. Appl. Energy 2016, 163, 190-200. [CrossRef]

69. Wi, Y.M.; Lee, J.U.; Joo, S.K. Electric Vehicle Charging Method for Smart Homes/Buildings with a Photovoltaic System. IEEE Trans. Consum. Electron. 2013, 59, 323-328. [CrossRef]

70. Dib, W.; Chasse, A.; Moulin, P.; Sciarretta, A.; Corde, G. Optimal Energy Management for an Electric Vehicle in Eco-Driving Applications. Control Eng. Pract. 2014, 29, 299-307. [CrossRef]

71. Tani, A.; Camara, M.B.; Dakyo, B. Energy Management Based on Frequency Approach for Hybrid Electric Vehicle Applications: Fuel-Cell/Lithium-Battery and Ultracapacitors. IEEE Trans. Veh. Technol. 2012, 61, 3375-3386. [CrossRef]

72. Wang, X.; He, H.; Sun, F.; Zhang, J. Application Study on the Dynamic Programming Algorithm for Energy Management of Plug-in Hybrid Electric Vehicles. Energies 2015, 8, 3225-3244. [CrossRef]

73. Song, Z.; Li, J.; Hou, J.; Hofmann, H.; Ouyang, M.; Du, J. The Battery-Supercapacitor Hybrid Energy Storage System in Electric Vehicle Applications: A Case Study. Energy 2018, 154, 433-441. [CrossRef]

74. Ali, M.U.; Zafar, A.; Nengroo, S.H.; Hussain, S.; Alvi, M.J.; Kim, H.-J. Towards a Smarter Battery Management System for Electric Vehicle Applications: A Critical Review of Lithium-Ion Battery State of Charge Estimation. Energies 2019, 12, 446. [CrossRef]

75. Kumar, M.S.; Revankar, S.T. Development Scheme and Key Technology of an Electric Vehicle: An Overview. Renew. Sustain. Energy Rev. 2017, 70, 1266-1285. [CrossRef]

76. Chen, Z.; Xia, B.; You, C.; Mi, C.C. A Novel Energy Management Method for Series Plug-in Hybrid Electric Vehicles. Appl. Energy 2015, 145, 172-179. [CrossRef]

77. Nojavan, S.; Zare, K.; Mohammadi-Ivatloo, B. Application of Fuel Cell and Electrolyzer as Hydrogen Energy Storage System in Energy Management of Electricity Energy Retailer in the Presence of the Renewable Energy Sources and Plug-in Electric Vehicles. Energy Convers. Manag. 2017, 136, 404-417. [CrossRef]

78. Yu, H.; Kuang, M.; McGee, R. Trip-Oriented Energy Management Control Strategy for Plug-in Hybrid Electric Vehicles. IEEE Trans. Control Syst. Technol. 2014, 22, 1323-1336. [CrossRef]

79. Falvo, M.C.; Lamedica, R.; Bartoni, R.; Maranzano, G. Energy Management in Metro-Transit Systems: An Innovative Proposal toward an Integrated and Sustainable Urban Mobility System Including Plug-in Electric Vehicles. Electr. Power Syst. Res. 2011, 81, 2127-2138. [CrossRef]

80. Zhao, D.; Stobart, R.; Dong, G.; Winward, E. Real-Time Energy Management for Diesel Heavy Duty Hybrid Electric Vehicles. IEEE Trans. Control Syst. Technol. 2015, 23, 829-841. [CrossRef]

81. Liu, G.; Ouyang, M.; Lu, L.; Li, J.; Hua, J. A Highly Accurate Predictive-Adaptive Method for Lithium-Ion Battery Remaining Discharge Energy Prediction in Electric Vehicle Applications. Appl. Energy 2015, 149, 297-314. [CrossRef]

82. Liu, T.; Tang, X.; Wang, H.; Yu, H.; Hu, X. Adaptive Hierarchical Energy Management Design for a Plug-In Hybrid Electric Vehicle. IEEE Trans. Veh. Technol. 2019, 68, 11513-11522. [CrossRef]

83. Mahmud, K.; Town, G.E. A Review of Computer Tools for Modeling Electric Vehicle Energy Requirements and Their Impact on Power Distribution Networks. Appl. Energy 2016, 172, 337-359. [CrossRef]

84. Zheng, C.; Li, W.; Liang, Q. An Energy Management Strategy of Hybrid Energy Storage Systems for Electric Vehicle Applications. IEEE Trans. Sustain. Energy 2018, 9, 1880-1888. [CrossRef]

85. Trovao, J.P.F.; Roux, M.A.; Menard, E.; Dubois, M.R. Energy- and Power-Split Management of Dual Energy Storage System for a Three-Wheel Electric Vehicle. IEEE Trans. Veh. Technol. 2017, 66, 5540-5550. [CrossRef]

86. Wang, X.; He, H.; Sun, F.; Sun, X.; Tang, H. Comparative Study on Different Energy Management Strategies for Plug-In Hybrid Electric Vehicles. Energies 2013, 6, 5656-5675. [CrossRef]

87. Wan, J.; Yan, H.; Li, D.; Zhou, K.; Zeng, L. Cyber-Physical Systems for Optimal Energy Management Scheme of Autonomous Electric Vehicle. Comput. J. 2013, 56, 947-956. [CrossRef]

88. Marzougui, H.; Kadri, A.; Martin, J.P.; Amari, M.; Pierfederici, S.; Bacha, F. Implementation of Energy Management Strategy of Hybrid Power Source for Electrical Vehicle. Energy Convers. Manag. 2019, 195, 830-843. [CrossRef]

89. Xu, B.; Rathod, D.; Zhang, D.; Yebi, A.; Zhang, X.; Li, X.; Filipi, Z. Parametric Study on Reinforcement Learning Optimized Energy Management Strategy for a Hybrid Electric Vehicle. Appl. Energy 2020, 259, 114200. [CrossRef]

90. Zhang, Q.; Li, G. Experimental Study on a Semi-Active Battery-Supercapacitor Hybrid Energy Storage System for Electric Vehicle Application. IEEE Trans. Power Electron. 2020, 35, 1014-1021. [CrossRef]

91. Ramadan, H.S.; Becherif, M.; Claude, F. Energy Management Improvement of Hybrid Electric Vehicles via Combined GPS/RuleBased Methodology. IEEE Trans. Autom. Sci. Eng. 2017, 14, 586-597. [CrossRef]

92. Shankar, R.; Marco, J.; Assadian, F. The Novel Application of Optimization and Charge Blended Energy Management Control for Component Downsizing within a Plug-in Hybrid Electric Vehicle. Energies 2012, 5, 4892-4923. [CrossRef]

93. Sarmah, S.B.; Kalita, P.; Garg, A.; Niu, X.; Zhang, X.-W.; Peng, X.; Bhattacharjee, D. A Review of State of Health Estimation of Energy Storage Systems: Challenges and Possible Solutions for Futuristic Applications of Li-Ion Battery Packs in Electric Vehicles. J. Electrochem. Energy Convers. Storage 2019, 16, 40801. [CrossRef]

94. Li, Y.; He, H.; Peng, J.; Wang, H. Deep Reinforcement Learning-Based Energy Management for a Series Hybrid Electric Vehicle Enabled by History Cumulative Trip Information. IEEE Trans. Veh. Technol. 2019, 68, 7416-7430. [CrossRef]

95. Zhang, Q.; Deng, W. An Adaptive Energy Management System for Electric Vehicles Based on Driving Cycle Identification and Wavelet Transform. Energies 2016, 9, 341. [CrossRef] 
96. Javorski Eckert, J.; Corrêa de Alkmin e Silva, L.; Mazzariol Santiciolli, F.; dos Santos Costa, E.; Corrêa, F.C.; Giuseppe Dedini, F. Energy Storage and Control Optimization for an Electric Vehicle. Int. J. Energy Res. 2018, 42, 3506-3523. [CrossRef]

97. Djerioui, A.; Houari, A.; Zeghlache, S.; Saim, A.; Benkhoris, M.F.; Mesbahi, T.; Machmoum, M. Energy Management Strategy of Supercapacitor/Fuel Cell Energy Storage Devices for Vehicle Applications. Int. J. Hydrogen Energy 2019, 44, 23416-23428. [CrossRef]

98. Zhou, Y.; Li, H.; Ravey, A.; Péra, M.C. An Integrated Predictive Energy Management for Light-Duty Range-Extended Plug-in Fuel Cell Electric Vehicle. J. Power Sources 2020, 451, 227780. [CrossRef]

99. Soumeur, M.A.; Gasbaoui, B.; Abdelkhalek, O.; Ghouili, J.; Toumi, T.; Chakar, A. Comparative Study of Energy Management Strategies for Hybrid Proton Exchange Membrane Fuel Cell Four Wheel Drive Electric Vehicle. J. Power Sources 2020, $462,228167$. [CrossRef]

100. Liu, Y.; Gao, J.; Qin, D.; Zhang, Y.; Lei, Z. Rule-Corrected Energy Management Strategy for Hybrid Electric Vehicles Based on Operation-Mode Prediction. J. Clean. Prod. 2018, 188, 796-806. [CrossRef]

101. Perullo, C.; Mavris, D. A Review of Hybrid-Electric Energy Management and Its Inclusion in Vehicle Sizing. Aircr. Eng. Aerosp. Technol. Int. J. 2014, 86, 550-557. [CrossRef]

102. Yang, B.; Wang, J.; Zhang, X.; Wang, J.; Shu, H.; Li, S.; He, T.; Lan, C.; Yu, T. Applications of Battery/Supercapacitor Hybrid Energy Storage Systems for Electric Vehicles Using Perturbation Observer Based Robust Control. J. Power Sources 2020, 448, 227444. [CrossRef]

103. Rezaei, A.; Burl, J.B.; Solouk, A.; Zhou, B.; Rezaei, M.; Shahbakhti, M. Catch Energy Saving Opportunity (CESO), an Instantaneous Optimal Energy Management Strategy for Series Hybrid Electric Vehicles. Appl. Energy 2017, 208, 655-665. [CrossRef]

104. Guo, N.; Shen, J.; Xiao, R.; Yan, W.; Chen, Z. Energy Management for Plug-in Hybrid Electric Vehicles Considering Optimal Engine ON/OFF Control and Fast State-of-Charge Trajectory Planning. Energy 2018, 163, 457-474. [CrossRef]

105. Florescu, A.; Bratcu, A.I.; Munteanu, I.; Rumeau, A.; Bacha, S. LQG Optimal Control Applied to On-Board Energy Management System of All-Electric Vehicles. IEEE Trans. Control Syst. Technol. 2015, 23, 1427-1439. [CrossRef]

106. Rezzak, D.; Boudjerda, N. Management and Control Strategy of a Hybrid Energy Source Fuel Cell/Supercapacitor in Electric Vehicles. Int. Trans. Electr. Energy Syst. 2017, 27, e2308. [CrossRef]

107. Zeynali, S.; Rostami, N.; Ahmadian, A.; Elkamel, A. Two-Stage Stochastic Home Energy Management Strategy Considering Electric Vehicle and Battery Energy Storage System: An ANN-Based Scenario Generation Methodology. Sustain. Energy Technol. Assess. 2020, 39, 100722. [CrossRef]

108. Zhang, Y.; Chu, L.; Fu, Z.; Xu, N.; Guo, C.; Zhao, D.; Ou, Y.; Xu, L. Energy Management Strategy for Plug-in Hybrid Electric Vehicle Integrated with Vehicle-Environment Cooperation Control. Energy 2020, 197, 117192. [CrossRef]

109. Hussain, S.; Ali, M.U.; Park, G.-S.; Nengroo, S.H.; Khan, M.A.; Kim, H.-J. A Real-Time Bi-Adaptive Controller-Based Energy Management System for Battery-Supercapacitor Hybrid Electric Vehicles. Energies 2019, 12, 4662. [CrossRef]

110. Ruan, J.; Song, Q.; Yang, W. The Application of Hybrid Energy Storage System with Electrified Continuously Variable Transmission in Battery Electric Vehicle. Energy 2019, 183, 315-330. [CrossRef]

111. Liu, C.; Murphey, Y.L. Optimal Power Management Based on Q-Learning and Neuro-Dynamic Programming for Plug-in Hybrid Electric Vehicles. IEEE Trans. Neural Netw. Learn. Syst. 2020, 31, 1942-1954. [CrossRef] [PubMed]

112. Lujan, J.M.; Guardiola, C.; Pla, B.; Reig, A. Analytical Optimal Solution to the Energy Management Problem in Series Hybrid Electric Vehicles. IEEE Trans. Veh. Technol. 2018, 67, 6803-6813. [CrossRef]

113. Wu, Y.; Zhang, Y.; Li, G.; Shen, J.; Chen, Z.; Liu, Y. A Predictive Energy Management Strategy for Multi-Mode Plug-in Hybrid Electric Vehicles Based on Multi Neural Networks. Energy 2020, 208, 118366. [CrossRef]

114. Bayat, P.; Baghramian, A.; Bayat, P. Implementation of Hybrid Electric Vehicle Energy Management System for Two Input Power Sources. J. Energy Storage 2018, 17, 423-440. [CrossRef]

115. Aoun, A.; Ibrahim, H.; Ghandour, M.; Ilinca, A. Supply Side Management vs. Demand Side Management of a Residential Microgrid Equipped with an Electric Vehicle in a Dual Tariff Scheme. Energies 2019, 12, 4351. [CrossRef]

116. Wang, C.; Huang, B.; Xu, W. An Integrated Energy Management Strategy with Parameter Match Method for Plug-in Hybrid Electric Vehicles. IEEE Access 2018, 6, 62204-62214. [CrossRef]

117. Boehme, T.J.; Held, F.; Rollinger, C.; Rabba, H.; Schultalbers, M.; Lampe, B. Application of an Optimal Control Problem to a Trip-Based Energy Management for Electric Vehicles. SAE Int. J. Altern. Powertrains 2013, 2, 115-126. [CrossRef]

118. Wang, C.; Yang, R.; Yu, Q. Wavelet Transform Based Energy Management Strategies for Plug-in Hybrid Electric Vehicles Considering Temperature Uncertainty. Appl. Energy 2019, 256, 113928. [CrossRef]

119. Fekri, S.; Assadian, F. The Design and Development of Multivariable Controls with the Application for Energy Management of Hybrid Electric Vehicles. Int. J. Veh. Des. 2012, 60, 225-247. [CrossRef]

120. Chen, Z.; Hu, H.; Wu, Y.; Zhang, Y.; Li, G.; Liu, Y. Stochastic Model Predictive Control for Energy Management of Power-Split Plug-in Hybrid Electric Vehicles Based on Reinforcement Learning. Energy 2020, 211, 118931. [CrossRef]

121. Cen, J.; Jiang, F. Li-Ion Power Battery Temperature Control by a Battery Thermal Management and Vehicle Cabin Air Conditioning Integrated System. Energy Sustain. Dev. 2020, 57, 141-148. [CrossRef]

122. Hmidi, M.E.; Salem, I.B.; Amraoui, L.E. An Efficient Method for Energy Management Optimization Control: Minimizing Fuel Consumption for Hybrid Vehicle Applications. Trans. Inst. Meas. Control 2019, 42, 69-80. [CrossRef] 
123. Badji, A.; Abdeslam, D.O.; Becherif, M.; Eltoumi, F.; Benamrouche, N. Analyze and Evaluate of Energy Management System for Fuel Cell Electric Vehicle Based on Frequency Splitting. Math. Comput. Simul. 2020, 167, 65-77. [CrossRef]

124. Sami, B.; Sihem, N.; Gherairi, S.; Adnane, C. A Multi-Agent System for Smart Energy Management Devoted to Vehicle Applications: Realistic Dynamic Hybrid Electric System Using Hydrogen as a Fuel. Energies 2019, 12, 474. [CrossRef]

125. Bernagozzi, M.; Charmer, S.; Georgoulas, A.; Malavasi, I.; Michè, N.; Marengo, M. Lumped Parameter Network Simulation of a Loop Heat Pipe for Energy Management Systems in Full Electric Vehicles. Appl. Therm. Eng. 2018, 141, 617-629. [CrossRef]

126. Amini, M.R.; Kolmanovsky, I.; Sun, J. Hierarchical MPC for Robust Eco-Cooling of Connected and Automated Vehicles and Its Application to Electric Vehicle Battery Thermal Management. IEEE Trans. Control Syst. Technol. 2021, 29, 316-328. [CrossRef]

127. Zhu, T.; Lot, R.; Wills, R.G.A.; Yan, X. Sizing a Battery-Supercapacitor Energy Storage System with Battery Degradation Consideration for High-Performance Electric Vehicles. Energy 2020, 208, 118336. [CrossRef]

128. Liu, Y.; Liu, J.; Qin, D.; Li, G.; Chen, Z.; Zhang, Y. Online Energy Management Strategy of Fuel Cell Hybrid Electric Vehicles Based on Rule Learning. J. Clean. Prod. 2020, 260, 121017. [CrossRef]

129. Xu, Q.; Mao, Y.; Zhao, M.; Cui, S. A Hybrid Electric Vehicle Dynamic Optimization Energy Management Strategy Based on a Compound-Structured Permanent-Magnet Motor. Energies 2018, 11, 2212. [CrossRef]

130. Li, W.; Xu, C.; Yu, H.; Gu, Y.; He, X. Energy Management with Dual Droop plus Frequency Dividing Coordinated Control Strategy for Electric Vehicle Applications. J. Mod. Power Syst. Clean Energy 2015, 3, 212-220. [CrossRef]

131. Li, T.; Liu, H.; Wang, H.; Yao, Y. Hierarchical Predictive Control-Based Economic Energy Management for Fuel Cell Hybrid Construction Vehicles. Energy 2020, 198, 117327. [CrossRef]

132. Mehrabi, A.; Nunna, H.S.V.S.K.; Dadlani, A.; Moon, S.; Kim, K. Decentralized Greedy-Based Algorithm for Smart Energy Management in Plug-in Electric Vehicle Energy Distribution Systems. IEEE Access 2020, 8, 75666-75681. [CrossRef]

133. Xue, Q.; Zhang, X.; Teng, T.; Zhang, J.; Feng, Z.; Lv, Q. A Comprehensive Review on Classification, Energy Management Strategy, and Control Algorithm for Hybrid Electric Vehicles. Energies 2020, 13, 5355. [CrossRef]

134. Yao, G.; Du, C.; Ge, Q.; Jiang, H.; Wang, Y.; Ait-Ahmed, M.; Moreau, L. Traffic-Condition-Prediction-Based HMA-FIS EnergyManagement Strategy for Fuel-Cell Electric Vehicles. Energies 2019, 12, 4426. [CrossRef]

135. Liu, G.; Zhang, J. An Energy Management of Plug-in Hybrid Electric Vehicles Based on Driver Behavior and Road Information. J. Intell. Fuzzy Syst. 2017, 33, 3009-3020. [CrossRef]

136. Mesbahi, T.; Bartholomeus, P.; Rizoug, N.; Sadoun, R.; Khenfri, F.; Le Moigne, P. Advanced Model of Hybrid Energy Storage System Integrating Lithium-Ion Battery and Supercapacitor for Electric Vehicle Applications. IEEE Trans. Ind. Electron. 2021, 68, 3962-3972. [CrossRef]

137. Su, Y.-D.; Preger, Y.; Burroughs, H.; Sun, C.; Ohodnicki, P.R. Fiber Optic Sensing Technologies for Battery Management Systems and Energy Storage Applications. Sensors 2021, 21, 1397. [CrossRef]

138. Zand, M.; Nasab, M.A.; Sanjeevikumar, P.; Maroti, P.K.; Holm-Nielsen, J.B. Energy Management Strategy for Solid-State Transformer-Based Solar Charging Station for Electric Vehicles in Smart Grids. IET Renew. Power Gener. 2020, 14, 3843-3852. [CrossRef]

139. de Melo, R.R.; Tofoli, F.L.; Daher, S.; Antunes, F.L.M. Interleaved Bidirectional DC-DC Converter for Electric Vehicle Applications Based on Multiple Energy Storage Devices. Electr. Eng. 2020, 102, 2011-2023. [CrossRef]

140. Demircali, A.; Koroglu, S. Jaya Algorithm-Based Energy Management System for Battery- and Ultracapacitor-Powered Ultralight Electric Vehicle. Int. J. Energy Res. 2020, 44, 4977-4985. [CrossRef]

141. Rashedi, M.; Mohammadian, M. Online Energy Management Applied to Fuel Cell Hybrid Electric Vehicles. Int. J. Electr. Hybrid Veh. 2010, 2, 315-328. [CrossRef]

142. Rahman, A.U.; Zehra, S.S.; Ahmad, I.; Armghan, H. Fuzzy Supertwisting Sliding Mode-Based Energy Management and Control of Hybrid Energy Storage System in Electric Vehicle Considering Fuel Economy. J. Energy Storage 2021, 37, 102468. [CrossRef]

143. Deng, Y.S.Y.; Li, H. Study of Bidirectional DC-DC Converter Interfacing Energy Storage for Vehicle Power Management Using Real Time Digital Simulator (RTDS). J. Power Electron. 2011, 11, 479-489. [CrossRef]

144. Vodovozov, V.; Raud, Z.; Petlenkov, E. Review on Braking Energy Management in Electric Vehicles. Energies $2021,14,4477$. [CrossRef]

145. Torabi, R.; Gomes, Á.; Morgado-Dias, F. Energy Transition on Islands with the Presence of Electric Vehicles: A Case Study for Porto Santo. Energies 2021, 14, 3439. [CrossRef]

146. Rehman, U.; Feng, D.; Su, H.; Numan, M.; Abbas, F. Network Overloading Management by Exploiting the In-System Batteries of Electric Vehicles. Int. J. Energy Res. 2021, 45, 5866-5880. [CrossRef]

147. Wang, L.; Wu, Z.; Cao, C. Integrated Optimization of Routing and Energy Management for Electric Vehicles in Delivery Scheduling. Energies 2021, 14, 1762. [CrossRef]

148. Xu, X.; Hu, W.; Liu, W.; Du, Y.; Huang, R.; Huang, Q.; Chen, Z. Risk Management Strategy for a Renewable Power Supply System in Commercial Buildings Considering Thermal Comfort and Stochastic Electric Vehicle Behaviors. Energy Convers. Manag. 2021, 230, 113831. [CrossRef]

149. Xiao, B.; Ruan, J.; Yang, W.; Walker, P.D.; Zhang, N. A Review of Pivotal Energy Management Strategies for Extended Range Electric Vehicles. Renew. Sustain. Energy Rev. 2021, 149, 111194. [CrossRef]

150. Yang, S.; Zhou, S.; Zhou, X.; Chen, F.; Li, Q.; Lu, Y.; Hua, Y.; Deng, H. Essential Technologies on the Direct Cooling Thermal Management System for Electric Vehicles. Int. J. Energy Res. 2021, 45, 14436-14464. [CrossRef] 
151. Zhu, T.; Wills, R.G.A.; Lot, R.; Ruan, H.; Jiang, Z. Adaptive Energy Management of a Battery-Supercapacitor Energy Storage System for Electric Vehicles Based on Flexible Perception and Neural Network Fitting. Appl. Energy 2021, 292, 116932. [CrossRef]

152. Zou, R.; Fan, L.; Dong, Y.; Zheng, S.; Hu, C. DQL Energy Management: An Online-Updated Algorithm and Its Application in Fix-Line Hybrid Electric Vehicle. Energy 2021, 225, 120174. [CrossRef]

153. Polverino, P.; Arsie, I.; Pianese, C. Optimal Energy Management for Hybrid Electric Vehicles Based on Dynamic Programming and Receding Horizon. Energies 2021, 14, 3502. [CrossRef]

154. Sellali, M.; Betka, A.; Djerdir, A.; Yang, Y.; Bahri, I.; Drid, S. A Novel Energy Management Strategy in Electric Vehicle Based on Ho Self-Gain Scheduled for Linear Parameter Varying Systems. IEEE Trans. Energy Convers. 2021, 36, 767-778. [CrossRef]

155. Piperidis, S.; Chrysomallis, I.; Georgakopoulos, S.; Ghionis, N.; Doitsidis, L.; Tsourveloudis, N. A ROS-Based Energy Management System for a Prototype Fuel Cell Hybrid Vehicle. Energies 2021, 14, 1964. [CrossRef]

156. Xu, H.; Shen, M. The Control of Lithium-Ion Batteries and Supercapacitors in Hybrid Energy Storage Systems for Electric Vehicles: A Review. Int. J. Energy Res. 2021, 45, 20524. [CrossRef]

157. Chen, L.; Ma, X.; Wei, S.; Yuan, D. Real-Time Energy Management Strategy of Multi-Wheel Electric Drive Vehicles with Load Power Prediction Function. IEEE Access 2021, 9, 20681-20694. [CrossRef]

158. Deng, T.; Tang, P.; Luo, J. A Novel Real-Time Energy Management Strategy for Plug-in Hybrid Electric Vehicles Based on Equivalence Factor Dynamic Optimization Method. Int. J. Energy Res. 2021, 45, 626-641. [CrossRef]

159. Lipu, M.S.H.; Faisal, M.; Ansari, S.; Hannan, M.A.; Karim, T.F.; Ayob, A.; Hussain, A.; Miah, M.S.; Saad, M.H.M. Review of Electric Vehicle Converter Configurations, Control Schemes and Optimizations: Challenges and Suggestions. Electronics 2021, 10, 477. [CrossRef]

160. Hannan, M.A.; How, D.N.T.; Mansor, M.; Hossain Lipu, M.S.; Ker, P.; Muttaqi, K. State-of-Charge Estimation of Li-Ion Battery Using Gated Recurrent Unit with One-Cycle Learning Rate Policy. IEEE Trans. Ind. Appl. 2021, 57, 2964-2971. [CrossRef]

161. Hannan, M.A.; How, D.N.T.; Hossain Lipu, M.S.; Ker, P.J.; Dong, Z.Y.; Mansur, M.; Blaabjerg, F. SOC Estimation of Li-Ion Batteries with Learning Rate-Optimized Deep Fully Convolutional Network. IEEE Trans. Power Electron. 2021, 36, 7349-7353. [CrossRef]

162. Hannan, M.A.; How, D.N.T.; Lipu, M.S.H.; Mansor, M.; Ker, P.J.; Dong, Z.Y.; Sahari, K.S.M.; Tiong, S.K.; Muttaqi, K.M.; Mahlia, T.M.I.; et al. Deep Learning Approach towards Accurate State of Charge Estimation for Lithium-Ion Batteries Using Self-Supervised Transformer Model. Sci. Rep. 2021, 11, 1-13. [CrossRef]

163. Mali, V.; Saxena, R.; Kumar, K.; Kalam, A.; Tripathi, B. Review on Battery Thermal Management Systems for Energy-Efficient Electric Vehicles. Renew. Sustain. Energy Rev. 2021, 151, 111611. [CrossRef]

164. Liu, H.; Wei, Z.; He, W.; Zhao, J. Thermal Issues about Li-Ion Batteries and Recent Progress in Battery Thermal Management Systems: A Review. Energy Convers. Manag. 2017, 150, 304-330. [CrossRef]

165. Gholami, M.; Sanjari, M.J. Multiobjective Energy Management in Battery-Integrated Home Energy Systems. Renew. Energy 2021, 177, 967-975. [CrossRef]

166. Herrmann, F.; Rothfuss, F. Introduction to Hybrid Electric Vehicles, Battery Electric Vehicles, and off-Road Electric Vehicles. Adv. Battery Technol. Electr. Veh. 2015, 3-16. [CrossRef]

167. Omar, N.; Daowd, M.; Bossche, P.V.D.; Hegazy, O.; Smekens, J.; Coosemans, T.; van Mierlo, J. Rechargeable Energy Storage Systems for Plug-in Hybrid Electric Vehicles-Assessment of Electrical Characteristics. Energies 2012, 5, 2952-2988. [CrossRef]

168. Nakamura, N.; Yokoshima, T.; Nara, H.; Momma, T.; Osaka, T. Suppression of Polysulfide Dissolution by Polypyrrole Modification of Sulfur-Based Cathodes in Lithium Secondary Batteries. J. Power Sources 2015, 274, 1263-1266. [CrossRef]

169. Arie, A.A.; Song, J.O.; Lee, J.K. Structural and Electrochemical Properties of Fullerene-Coated Silicon Thin Film as Anode Materials for Lithium Secondary Batteries. Mater. Chem. Phys. 2009, 113, 249-254. [CrossRef]

170. Teng, J.H.; Luan, S.W.; Lee, D.J.; Huang, Y.Q. Optimal Charging/Discharging Scheduling of Battery Storage Systems for Distribution Systems Interconnected with Sizeable PV Generation Systems. IEEE Trans. Power Syst. 2013, 28, 1425-1433. [CrossRef]

171. Zhang, T.; Chen, W.; Han, Z.; Cao, Z. Charging Scheduling of Electric Vehicles with Local Renewable Energy under Uncertain Electric Vehicle Arrival and Grid Power Price. IEEE Trans. Veh. Technol. 2014, 63, 2600-2612. [CrossRef]

172. Han, X.; Ouyang, M.; Lu, L.; Li, J. Simplification of Physics-Based Electrochemical Model for Lithium Ion Battery on Electric Vehicle. Part II: Pseudo-Two-Dimensional Model Simplification and State of Charge Estimation. J. Power Sources 2015, 278, 814-825. [CrossRef]

173. Bocklisch, T. Hybrid Energy Storage Systems for Renewable Energy Applications. Energy Procedia 2015, 73, 103-111. [CrossRef]

174. Arcos-Aviles, D.; Pascual, J.; Marroyo, L.; Sanchis, P.; Guinjoan, F. Fuzzy Logic-Based Energy Management System Design for Residential Grid-Connected Microgrids. IEEE Trans. Smart Grid 2018, 9, 530-543. [CrossRef]

175. Lopez-Sanz, J.; Ocampo-Martinez, C.; Alvarez-Florez, J.; Moreno-Eguilaz, M.; Ruiz-Mansilla, R.; Kalmus, J.; Gräeber, M.; Lux, G. Nonlinear Model Predictive Control for Thermal Management in Plug-in Hybrid Electric Vehicles. IEEE Trans. Veh. Technol. 2017, 66, 3632-3644. [CrossRef]

176. Bracco, S.; Delfino, F.; Pampararo, F.; Robba, M.; Rossi, M. A Dynamic Optimization-Based Architecture for Polygeneration Microgrids with Tri-Generation, Renewables, Storage Systems and Electrical Vehicles. Energy Convers. Manag. 2015, 96, 511-520. [CrossRef]

177. Akar, F.; Tavlasoglu, Y.; Ugur, E.; Vural, B.; Aksoy, I. A Bidirectional Nonisolated Multi-Input DC-DC Converter for Hybrid Energy Storage Systems in Electric Vehicles. IEEE Trans. Veh. Technol. 2016, 65, 7944-7955. [CrossRef] 
178. Moura, S.J.; Stein, J.L.; Fathy, H.K. Battery-Health Conscious Power Management in Plug-in Hybrid Electric Vehicles via Electrochemical Modeling and Stochastic Control. IEEE Trans. Control Syst. Technol. 2013, 21, 679-694. [CrossRef]

179. Abbatantuono, G.; Bruno, S.; Scala, L.A.; Sbrizzai, R.; Stecchi, U. Power Flow Control in Electric Distribution Systems Integrating Storage Devices. Int. J. Power Syst. 2016, 1, 78-83.

180. Park, H. A Design of Air Flow Configuration for Cooling Lithium Ion Battery in Hybrid Electric Vehicles. J. Power Sources 2013, 239, 30-36. [CrossRef]

181. Hannan, M.A.; Lipu, M.S.H.; Hussain, A.; Ker, P.J.; Mahlia, T.M.I.; Mansor, M.; Ayob, A.; Saad, M.H.; Dong, Z.Y. Toward Enhanced State of Charge Estimation of Lithium-Ion Batteries Using Optimized Machine Learning Techniques. Sci. Rep. 2020, $10,1-15$. [CrossRef] [PubMed]

182. Hossain Lipu, M.S.; Hannan, M.A.; Hussain, A.; Ayob, A.; Saad, M.H.M.; Muttaqi, K.M. State of Charge Estimation in Lithium-Ion Batteries: A Neural Network Optimization Approach. Electronics 2020, 9, 1546. [CrossRef]

183. Hossain Lipu, M.S.; Hannan, M.A.; Hussain, A.; Ayob, A.; Saad, M.H.M.; Karim, T.F.; How, D.N.T. Data-Driven State of Charge Estimation of Lithium-Ion Batteries: Algorithms, Implementation Factors, Limitations and Future Trends. J. Clean. Prod. 2020, 277, 124110. [CrossRef]

184. Zhang, Y.; Liu, Y.; Huang, Y.; Chen, Z.; Li, G.; Hao, W.; Cunningham, G.; Early, J. An Optimal Control Strategy Design for Plug-in Hybrid Electric Vehicles Based on Internet of Vehicles. Energy 2021, 228, 120631. [CrossRef]

185. Chen, Z.; Zhao, H.; Shu, X.; Zhang, Y.; Shen, J.; Liu, Y. Synthetic State of Charge Estimation for Lithium-Ion Batteries Based on Long Short-Term Memory Network Modeling and Adaptive H-Infinity Filter. Energy 2021, 228, 120630. [CrossRef]

186. Shu, X.; Li, G.; Zhang, Y.; Shen, S.; Chen, Z.; Liu, Y. Stage of Charge Estimation of Lithium-Ion Battery Packs Based on Improved Cubature Kalman Filter with Long Short-Term Memory Model. IEEE Trans. Transp. Electrif. 2021, 7, 1271-1284. [CrossRef]

187. Liu, Y.; Li, J.; Gao, J.; Lei, Z.; Zhang, Y.; Chen, Z. Prediction of Vehicle Driving Conditions with Incorporation of Stochastic Forecasting and Machine Learning and a Case Study in Energy Management of Plug-in Hybrid Electric Vehicles. Mech. Syst. Signal Process. 2021, 158, 107765. [CrossRef]

188. Yusof, N.K.; Abas, P.E.; Mahlia, T.M.I.; Hannan, M.A. Techno-Economic Analysis and Environmental Impact of Electric Buses. World Electr. Veh. J. 2021, 12, 31. [CrossRef]

189. Faisal, M.; Hannan, M.A.; Ker, P.J.; Hossain Lipu, M.S.; Uddin, M.N. Fuzzy-Based Charging-Discharging Controller for LithiumIon Battery in Microgrid Applications. IEEE Trans. Ind. Appl. 2021, 57, 4187-4195. [CrossRef]

190. Touma, H.J.; Mansor, M.; Rahman, M.S.A.; Kumaran, V.; Mokhlis, H.B.; Ying, Y.J.; Hannan, M.A. Energy Management System of Microgrid: Control Schemes, Pricing Techniques, and Future Horizons. Int. J. Energy Res. 2021, 45, 12728-12739. [CrossRef]

191. Li, J.; Wu, X.; Xu, M.; Liu, Y. A Real-Time Optimization Energy Management of Range Extended Electric Vehicles for Battery Lifetime and Energy Consumption. J. Power Sources 2021, 498, 229939. [CrossRef]

192. Shaobo, X.; Qiankun, Z.; Xiaosong, H.; Yonggang, L.; Xianke, L. Battery Sizing for Plug-in Hybrid Electric Buses Considering Variable Route Lengths. Energy 2021, 226, 120368. [CrossRef]

193. Chen, Z.; Liu, Y.; Ye, M.; Zhang, Y.; Li, G. A Survey on Key Techniques and Development Perspectives of Equivalent Consumption Minimisation Strategy for Hybrid Electric Vehicles. Renew. Sustain. Energy Rev. 2021, 151, 111607. [CrossRef]

194. Akinlabi, A.A.H.; Solyali, D. Configuration, Design, and Optimization of Air-Cooled Battery Thermal Management System for Electric Vehicles: A Review. Renew. Sustain. Energy Rev. 2020, 125, 109815. [CrossRef]

195. Faggioli, E.; Rena, P.; Danel, V.; Andrieu, X.; Mallant, R.; Kahlen, H. Supercapacitors for the Energy Management of Electric Vehicles. J. Power Sources 1999, 84, 261-269. [CrossRef]

196. Thanapalan, K.; Zhang, F.; Premier, G.; Maddy, J.; Guwy, A. Energy Management Effects of Integrating Regenerative Braking into a Renewable Hydrogen Vehicle. In Proceedings of the 2012 UKACC International Conference on Control, CONTROL, Cardiff, UK, 3-5 September 2012; pp. 924-928. [CrossRef]

197. Yang, Y.P.; Guan, R.M.; Huang, Y.M. Hybrid Fuel Cell Powertrain for a Powered Wheelchair Driven by Rim Motors. J. Power Sources 2012, 212, 192-204. [CrossRef]

198. Lv, Y.; Yuan, H.; Liu, Y.; Wang, Q. Fuzzy Logic Based Energy Management Strategy of Battery-Ultracapacitor Composite Power Supply for HEV. In Proceedings of the 2010 1st International Conference on Pervasive Computing, Signal Processing and Applications, PCSPA, Harbin, China, 17-19 September 2010; pp. 1209-1214. [CrossRef]

199. Tarascon, J.-M.; Recham, N.; Armand, M.; Chotard, J.-N.; Barpanda, P.; Walker, W.; Dupont, L. Hunting for Better Li-Based Electrode Materials via Low Temperature Inorganic Synthesis. Chem. Mater. 2009, 22, 724-739. [CrossRef]

200. Hoque, M.M.; Hannan, M.A.; Mohamed, A. Optimal CC-CV Charging of Lithium-Ion Battery for Charge Equalization Controller. In Proceedings of the 2016 International Conference on Advances in Electrical, Electronic and Systems Engineering, ICAEES 2016, Putrajaya, Malaysia, 14-16 November 2017; pp. 610-615. [CrossRef]

201. Nikzad, M.R.; Radan, A. Accurate Loss Modelling of Fuel Cell Boost Converter and Traction Inverter for Efficiency Calculation in Fuel Cell-Battery Hybrid Vehicles. In Proceedings of the PEDSTC 2010-1st Power Electronics and Drive Systems and Technologies Conference, Tehran, Iran, 17-18 May 2010; pp. 218-223. [CrossRef]

202. Guilbert, D.; Mohammadi, A.; Gaillard, A.; N’Diaye, A.; Djerdir, A. Interactions between Fuel Cell and DC/DC Converter for Fuel Cell Electric Vehicle Applications: Influence of Faults. In Proceedings of the IECON Proceedings (Industrial Electronics Conference), Vienna, Austria, 10-13 November 2013; pp. 912-917. [CrossRef] 
203. Thounthong, P.; Pierfederici, S.; Martin, J.P.; Hinaje, M.; Davat, B. Modeling and Control of Fuel Cell/Supercapacitor Hybrid Source Based on Differential Flatness Control. IEEE Trans. Veh. Technol. 2010, 59, 2700-2710. [CrossRef]

204. Solano Martínez, J.; Hissel, D.; Péra, M.C.; Amiet, M. Practical Control Structure and Energy Management of a Testbed Hybrid Electric Vehicle. IEEE Trans. Veh. Technol. 2011, 60, 4139-4152. [CrossRef]

205. Hu, Y.; Yu, Y.; Huang, K.; Wang, L. Development Tendency and Future Response about the Recycling Methods of Spent Lithium-Ion Batteries Based on Bibliometrics Analysis. J. Energy Storage 2020, 27, 101111. [CrossRef]

206. Dunn, J.B.; Gaines, L.; Sullivan, J.; Wang, M.Q. Impact of Recycling on Cradle-to-Gate Energy Consumption and Greenhouse Gas Emissions of Automotive Lithium-Ion Batteries. Environ. Sci. Technol. 2012, 46, 12704-12710. [CrossRef] [PubMed]

207. Amarakoon, S.; Smith, J.; Segal, B. Application of Life-Cycle Assessment to Nanoscale Technology: Lithium-Ion Batteries for Electric Vehicles; United States Environmental Protection Agency: Washington, DC, USA, 2013.

208. Notter, D.A.; Gauch, M.; Widmer, R.; Wäger, P.; Stamp, A.; Zah, R.; Althaus, H.-J. Contribution of Li-Ion Batteries to the Environmental Impact of Electric Vehicles. Environ. Sci. Technol. 2010, 44, 6550-6556. [CrossRef]

209. Papadis, E.; Tsatsaronis, G. Challenges in the Decarbonization of the Energy Sector. Energy 2020, 205, 118025. [CrossRef]

210. Jägemann, C.; Fürsch, M.; Hagspiel, S.; Nagl, S. Decarbonizing Europe's Power Sector by 2050-Analyzing the Economic Implications of Alternative Decarbonization Pathways. Energy Econ. 2013, 40, 622-636. [CrossRef]

211. Child, M.; Kemfert, C.; Bogdanov, D.; Breyer, C. Flexible Electricity Generation, Grid Exchange and Storage for the Transition to a 100\% Renewable Energy System in Europe. Renew. Energy 2019, 139, 80-101. [CrossRef] 